\title{
The Cyanotoxin 2,4-DAB Reduces Viability and Causes Behavioral and Molecular Dysfunctions Associated with Neurodegeneration in Larval Zebrafish
}

\author{
Rubia M. Martin ${ }^{1} \cdot$ Michael S. Bereman $^{1} \cdot$ Kurt C. Marsden ${ }^{1}[0$
}

Received: 13 October 2021 / Revised: 30 November 2021 / Accepted: 16 December 2021 / Published online: 14 January 2022

(c) The Author(s) 2022

\begin{abstract}
Exposure to cyanotoxins has been linked to neurodegenerative diseases, including amyotrophic lateral sclerosis, Alzheimer's, and Parkinson's disease. While the cyanotoxin $\beta$-methylamino-L-alanine (BMAA) has received much attention, cyanobacteria produce many cyanotoxic compounds, several of which have been detected in nature alongside BMAA, including 2,4diaminobutyric acid (2,4-DAB) and N-(2-aminoethyl)glycine (AEG). Thus, the question of whether 2,4-DAB and AEG also cause neurotoxic effects in vivo is of great interest, as is the question of whether they interact to enhance toxicity. Here, we evaluate the toxic and neurotoxic effects of these cyanotoxins alone or in combination by measuring zebrafish larval viability and behavior after exposure. 2,4-DAB was the most potent cyanotoxin as it decreased larval viability by approximately $50 \%$ at 6 days post fertilization, while BMAA and AEG decreased viability by just $16 \%$ and $8 \%$, respectively. Although we only observed minor neurotoxic effects on spontaneous locomotion, BMAA and AEG enhanced acoustic startle sensitivity, and they interacted in an additive manner to exert their effects. 2,4-DAB; however, only modulated startle kinematics, an indication of motor dysfunction. To investigate the mechanisms of 2,4-DAB's effects, we analyzed the protein profile of larval zebrafish exposed to $500 \mu \mathrm{M} 2,4-\mathrm{DAB}$ at two time points and identified molecular signatures consistent with neurodegeneration, including disruption of metabolic pathways and downregulation of the ALS-associated genes SOD1 and UBQLN4. Together, our data demonstrate that BMAA and its isomers AEG and 2,4-DAB cause neurotoxic effects in vivo, with 2,4-DAB as the most potent of the three in the zebrafish model.
\end{abstract}

Keywords Cyanotoxins $\cdot$ Mixtures $\cdot 2,4-D A B \cdot$ Zebrafish $\cdot$ Behavior $\cdot$ Proteomics

\section{Introduction}

Exposure to BMAA ( $\beta$-N-methylamino-L-alanine), a noncanonical amino acid, has been epidemiologically associated with the onset of sporadic neurodegenerative diseases, including amyotrophic lateral sclerosis (ALS) (Banack and Cox 2003), Parkinson's (Nunes-Costa et al. 2020), and Alzheimer's diseases (Silva et al. 2020). BMAA is naturally released into the water by a diverse taxa of cyanobacteria, marine diatoms, and dinoflagellates (Cox et al. 2005; Réveillon et al. 2016), and it persists in the environment not only during and after a cyanobacteria bloom collapse (Réveillon et al. 2015) but also

Kurt C. Marsden

kcmarsde@ncsu.edu

1 Department of Biological Sciences, North Carolina State University, Raleigh, NC, USA in terrestrial ecosystems (Metcalf et al. 2012). BMAA can also bioaccumulate and be transferred to higher trophic levels ( $\mathrm{Li}$ et al. 2019). In vitro studies have shown that BMAA causes excitotoxicity in primary neuronal and glial cell cultures (Chiu et al. 2012, 2013), and it is also known to induce differential expression of genes and proteins associated with mitochondrial dysfunction and protein aggregation (Chiu et al. 2015; Beri et al. 2017). BMAA has also recently been suggested to act through alterations in gut microbiota (Nunes-Costa et al. 2020). These results suggest that BMAA may be a link to sporadic neuropathology; however, direct evidence that physiologically relevant levels of BMAA can induce neurodegeneration in animal models has been elusive (Karamyan and Speth 2008), although more recent work found that developmental exposure to BMAA in rats produced behavioral and pathological signs of neurodegeneration such as TDP-43 inclusions and hyperphosphorylated tau (Scott et al. 2017; Scott and Downing 2019). 
While laboratory studies have largely focused on BMAA in isolation, in the environment, it has been detected along with other cyanotoxins, including the BMAA isomers 2,4 diaminobutyric acid (2,4-DAB) and aminoethyl glycine (AEG) (Supplemental Fig. 1) (Metcalf et al. 2008; Réveillon et al. 2015; Chatziefthimiou et al. 2018). Variation in detection and analysis methods has led to discrepancies in the prevalence and levels of these cyanotoxins in environmental samples (reviewed in Faassen 2014), but more recent studies observed 2,4-DAB and AEG more frequently and at higher concentrations than BMAA (Violi et al. 2019; Vo Duy et al. 2019). The neurotoxicity of these isomers is relatively understudied, and because a natural exposure is likely to involve a combination of BMAA and other co-occurring cyanotoxins such as its isomers, it is particularly important to investigate the neurotoxic effects of exposure to cyanotoxic mixtures.

A preliminary binary mixture study showed synergistic neurotoxic effects of low concentrations of BMAA $(10-100 \mu \mathrm{M})$ and methylmercury $(3 \mu \mathrm{M})$ in primary cortical cell cultures (Rush et al. 2012). We have also previously demonstrated that BMAA can interact with its isomers 2,4-DAB and AEG in an in vitro system to enhance caspase activity and induce neurodegenerative processes in motor neuron-like NSC-34 cells (Martin et al. 2019). A recent in vitro study revealed that the isomer AEG is a more potent neurotoxin than BMAA and that the toxicity of AEG is mediated by the induction of free radicals as well as the activation of metabotropic glutamate receptors (i.e., mGluR5) (Schneider et al. 2020). Another in vitro study found that 2,4-DAB and BMAA exerted similar levels of toxicity in decreasing the viability of human neuroblastoma cells (Main et al. 2018). Like BMAA, 2,4-DAB also activates N-methyl-D-aspartate (NMDA) receptors (Spasic et al. 2018), a mode of action that is also a primary mechanism of BMAA-induced neurotoxicity (Lobner 2009). These in vitro studies recognize the neurotoxic potential of 2,4-DAB and AEG and highlight the importance of examining their individual and potential combined effects with BMAA in vivo. Here, we aimed to identify which individual cyanotoxic isomer and/or which combination of BMAA, AEG, and 2,4DAB elicits the greatest toxicity in larval zebrafish, using an axial simplex mixture design (Cornell 2011). We evaluated the viability of larval zebrafish after exposure to 10 different ratios of all three cyanotoxic components. We also examined behavioral indicators of neurotoxicity with a highthroughput behavior testing platform and found that there was little interaction between the toxins and that 2,4-DAB is a more potent toxin than either BMAA or AEG in vivo. To then identify early molecular changes that trigger toxicity of 2,4-DAB, we performed discovery proteomics at two developmental timepoints after exposure. Our results show that processes associated with neurodegeneration are impacted, including $\mathrm{Ca}^{2+}$ signaling, the unfolded protein response, and endoplasmic reticulum stress. Together, our data highlight the importance of studying mixtures and reveal 2,4-DAB to be a more potent neurotoxin than BMAA and AEG in the zebrafish model.

\section{Materials and Methods}

\section{Chemicals}

Synthetic BMAA, AEG, and 2,4-DAB standards were obtained from Sigma Aldrich (St. Louis, MO). Water, acetonitrile, methanol, acetic acid, and formic acid were all Optima LC-MS grade solvents purchased from Fisher Scientific (Tewksbury, MA, USA). Stock solutions of BMAA, AEG, and 2,4-DAB at $10 \mathrm{mg} \mathrm{mL}^{-1}$ were used for all mixture's dilutions. All dilutions were prepared in HPLC-grade water.

\section{Mixture Design}

Experiments were designed to address in vivo toxicity after exposure to BMAA, AEG, and 2,4-DAB alone or in combination, in which both variation in experiment replicates and reproducibility were assessed. To detect interactive effects in a three-component mixture, we used simplex axial design (Cornell 2011). Three compounds were studied at 7 different mixture combinations. The simplex axial design generally includes a test solution named center point in which all factors are studied at their equimolar concentrations and the linear average value between their maximum (1) and minimum (-1) levels, allowing for an accurate estimation of pure error, lack of fit (Cornell 2011). The mixture design was generated using Design Expert ${ }^{\circledR}$ software where each run was controlled to a total concentration of $500 \mu \mathrm{M}$. The $500 \mu \mathrm{M}$ concentration in which was chosen based on the lowest observable adverse effect level (LOAEL) found in our previous in vitro and in vivo dose response studies (Martin et al. 2020).

\section{Zebrafish Husbandry and Exposures}

All animal use and procedures were approved by the North Carolina State University IACUC. Zebrafish (Danio rerio) embryos from multiple crosses of wild-type tupfel longfin (TLF) strain adults were collected and placed into Petri dishes containing E3 medium, and unfertilized eggs were removed as described previously. Embryos from all clutches were mixed and randomly sorted into 24 well plates (8-10 animals per well) containing $1 \mathrm{~mL}$ of E3 per well.

At $6 \mathrm{~h}$ post fertilization (hpf), embryos were treated with vehicle, individual cyanotoxins, their binary mixtures, and their three-component mixtures (Fig. 1). All treatments were performed in triplicate and were repeated in each of 


\begin{tabular}{|c|c|c|c|c|c|c|}
\hline \multicolumn{4}{|c|}{ 2,4 DAB } & & & \\
\hline & & & 1 & 0 & 1 & 0 \\
\hline & & & 2 & 0.5 & 0.5 & 0 \\
\hline & & & 3 & 0.667 & 0.167 & 0.167 \\
\hline & & & 4 & 0.167 & 0.167 & 0.667 \\
\hline & 0 & & 5 & 0.5 & 0 & 0.5 \\
\hline & & & 6 & 0.167 & 0.667 & 0.167 \\
\hline & & & 7 & 1 & 0 & 0 \\
\hline & & & 8 & 0 & 0 & 1 \\
\hline & 0 & & 9 & 0 & 1 & 0 \\
\hline & & & 10 & 1 & 0 & 0 \\
\hline & & & 11 & 0.333 & 0.333 & 0.333 \\
\hline & 0 & & 12 & 0 & 0 & 1 \\
\hline AEG & & 2,4 DAB & 13 & 0 & 0.5 & 0 \\
\hline $500 \mu \mathrm{M}$ & & $500 \mu \mathrm{M}$ & 14 & 0.5 & 0.5 & 0 \\
\hline
\end{tabular}

B Exposures

Testing Apparatus

\section{Endpoints}

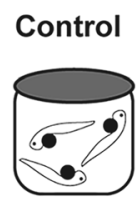

Individual Cyanotoxins
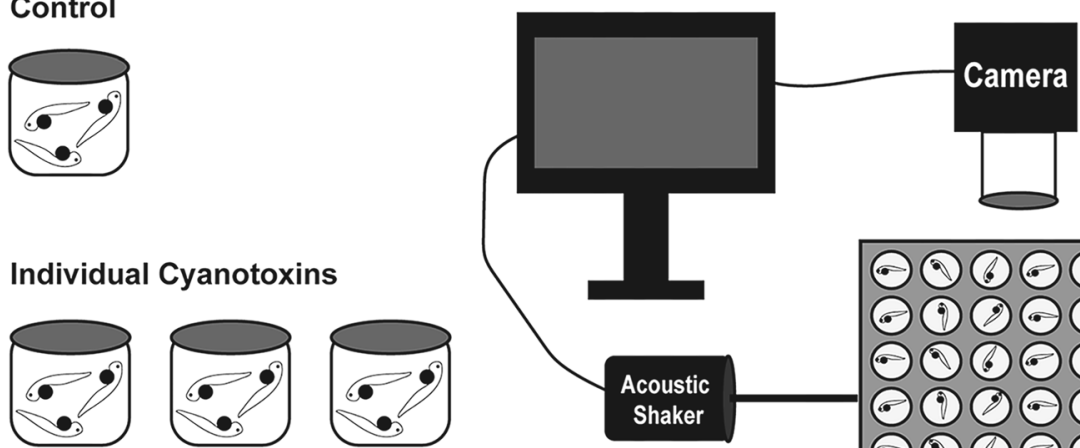

Viability Assesment

Across 6 Days of Exposure

\section{Binary Mixtures}
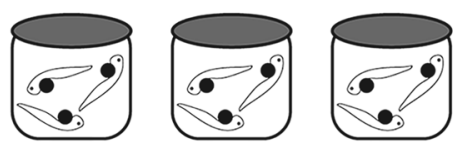

\section{2,4 DAB \\ $(500 \mu \mathrm{M})$}

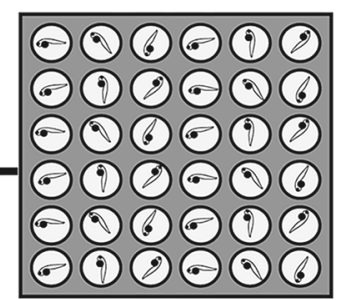

\section{Spontaneous Movement}

- Total Distance Traveled - Average Speed

Acoustic Startle

- Startle Sensitivity

- Startle Kinematics

Three Component Mixtures
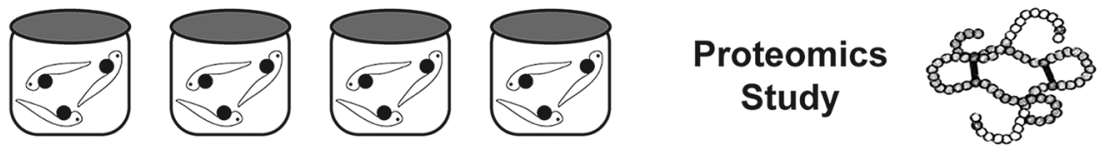

Molecular Signatures

- Proteins Regulation

- Canonical Pathways

-Upstream Regulators

Fig. 1 Experimental design. A 2D simplex axial design and randomized Design of Experiments (DoE) design grid representing the composition for each solution. B Exposure plan for larval zebrafish

2 separate experiments. Embryos were incubated at $29{ }^{\circ} \mathrm{C}$ on a $14 \mathrm{~h}: 10 \mathrm{~h}$ light-dark cycle, and $100 \%$ of the media was exchanged for fresh solutions daily. Embryos/larvae were from $6 \mathrm{~h}$ post fertilization (hpf) to 6 days post fertilization (dpf) followed by the high throughput behavior testing, proteomics analysis, and endpoints measurements for these studies

exposed to treatments up to 6 days post fertilization (dpf) for the behavior assays or up to 2 or $4 \mathrm{dpf}$ for the proteomics assays. 


\section{Zebrafish Viability and Behavior Assessment}

Mortality due to treatment toxicity was assessed daily and dead fish were counted and removed. During daily media changes, each fish was also assessed for a set of developmental phenotypes (pericardial edema, otic vesicle, pigmentation defects, small eyes, small heads, body axis defects such as curved or bent tails, and uninflated swim bladders), and any fish exhibiting these phenotypes was removed. Significant differences in viability data were analyzed via Kaplan-Meir survival analysis with Mantel-Cox Log-rank test using Prism 8.

\section{Behavior Assays and Analysis}

Prior to behavior testing, any dead fish or fish exhibiting developmental malformations such as pericardial edema, otic vesicle defects, pigmentation defects, small eyes, small heads, body axis defects such as curved or bent tails, and uninflated swim bladders were removed. Screened 6 dpf larvae were adapted to the testing lighting and temperature conditions for $30 \mathrm{~min}$ prior to testing. Behavior testing was done as previously described (Burgess and Granato 2007a, b; Marsden et al. 2018). Briefly, larvae were transferred to individual $9 \mathrm{~mm}$ round wells on a 36-well laser-cut acrylic testing grid. Larvae acclimated for $5 \mathrm{~min}$ and then spontaneous locomotor activity was recorded for $18.5 \mathrm{~min}$ at $640 \times 640 \mathrm{px}$ resolution at 50 frames per second (fps) using a Photron mini UX-50 high-speed camera. The same set of larvae were then presented with a total of 60 acoustic stimuli, 10 at each of 6 intensities $(13.6,25.7,29.2,35.5,39.6$, and $53.6 \mathrm{~dB}$ ), with a $20 \mathrm{~s}$ interstimulus interval (ISI). Startle responses were recorded at $1000 \mathrm{fps}$. Stimuli were delivered by an acoustic-vibrational shaker to which the testing grid was directly mounted. All stimuli were calibrated with a PCB Piezotronics accelerometer (\#355B04) and signal conditioner (\#482A21), and voltage outputs were converted to $d B$ using the formula $d B=20 \log V$. Analysis of recorded behaviors was done using FLOTE software as described previously (Marsden et al. 2018). Startle response C-bends were automatically identified using defined kinematic parameters. A startle sensitivity index was calculated for individual larvae by calculating the area under the curve of startle frequency versus stimulus intensity using Prism 8 software (GraphPad). Statistical analyses were performed using JMP pro 14 from SAS Institute, Cary, NC. Data were analyzed for effects between the groups (comparison of means), using Tukey-Kramer HSD, Alpha 0.05. Bar graphs were generated using Prism 8.

\section{Global Proteomics Study}

\section{The Cyanotoxin 2,4-DAB Exposure}

Zebrafish embryos from at least ten different fish pairs per batch were collected and randomized immediately after fertilization and transferred in groups of 10 into 24-well plates in E3 media. At $6 \mathrm{hpf}$, zebrafish embryos were treated with vehicle or 2,4-DAB at $500 \mu \mathrm{M}$ in E3. Embryos were incubated at $29^{\circ} \mathrm{C}$ on a $14 \mathrm{~h}: 10 \mathrm{~h}$ light-dark cycle, and $100 \%$ of the media was exchanged for fresh treated solutions daily. Embryos/larvae were exposed to 2,4-DAB until 2 or $4 \mathrm{dpf}$. Exactly ten screened larvae were pooled for each of 3 biological replicates for each condition and then flash frozen in liquid nitrogen and stored at $-80{ }^{\circ} \mathrm{C}$. Any dead fish or fish exhibiting developmental malformations such as pericardial edema, otolith malformations, pigmentation defects, small eyes, small heads, body axis defects such as curved or bent tails, and uninflated swim bladders were removed prior to proteomic analysis.

\section{Sample Preparation and LC MS/MS}

Details of sample preparation, protein extraction, and digestion via filter aided sample preparation (FASP) can be found in Supplemental Methods. Details regarding the LC-MS/MS data collection are also provided in the Supplemental Methods. Raw data files obtained in this experiment have been made available on the Chorus LC-MS data repository and can be assessed under the project ID\#1738.

\section{Proteomics Data Analysis}

Label-free quantification (LFQ) was performed in MaxQuant (version.1.5.60), which uses the Andromeda algorithm (Tyanova et al. 2016). Both dynamic (i.e., methionine oxidation and N-terminal acetylation) and fixed modifications (i.e., cysteine carbamido-methylation) were used for the database search along with a maximum of two missed cleavages. The minimum peptide length was set to 7 amino acids and the false discovery rate (FDR) for peptide and protein identification was set to 0.01 . All other search parameters were left as default values. Data were searched against the Danio rerio Swiss Prot protein database (\# protein sequences $=56281$, accessed 01/25/2021). Comparison of LFQ intensities across the whole set of measurements was investigated using Perseus software (version 1.5.1.6), where calculation of statistical significance was determined using two-way Student's $t$-test and FPR ( $p$ value $\leq 0.05)$. 


\section{Pathway Analysis}

Ingenuity Pathway Analysis (IPA) software was used to identify the function, specific processes, and enriched pathways of the differentially expressed proteins using the "Core Analysis" function. Only significantly differentially expressed proteins ( $p$ value $\leq 0.05$ ) were submitted to IPA. We used an empirical background protein database to evaluate the significance of pathway enrichment. The database was created by using all the proteins that were detected in our samples (Khatri and Drăghici 2005).

\section{Results}

An overview of the experiments in this study is illustrated in Fig. 1. An optimal mixture design was created using Design of Experiments (DoE) to establish the concentrations of BMAA, AEG, and 2,4-DAB to be used in the exposures. The 2D axial design generates a symmetrical triangle plot for a three-variable case, displaying 7 mixtures of different ratios along with each individual cyanotoxin. An additional 4 replicate tests were applied for a total of 14 runs to evaluate reproducibility and the lack of fit for the derived model (Fig. 1A). In brief, zebrafish larvae were exposed to BMAA, AEG, and 2,4-DAB as well as their binary/three-component mixture combinations from 6 hpf to $6 \mathrm{dpf}$. Viability of the larvae was assessed, and neurotoxicity of surviving zebrafish larvae was evaluated via two behavioral assays: spontaneous locomotion and acoustic startle response. To investigate perturbed molecular pathways associated with 2,4-DAB toxicity, the 2,4-DAB-exposed larvae were collected and subjected to discovery proteomics at two developmental stages: $2 \mathrm{dpf}$, prior to most toxin-induced mortality and $4 \mathrm{dpf}$, after toxin-induced mortality ceased (Fig. 1B).

\section{Viability Assessment: 2,4-DAB is More Toxic Than BMAA and AEG In Vivo}

To determine if exposure to cyanotoxins could induce organismal mortality, we aimed to evaluate the viability of zebrafish larvae during exposure to BMAA, AEG, and 2,4$\mathrm{DAB}$ alone or in combination. We exposed zebrafish larvae from $6 \mathrm{hpf}$ to $6 \mathrm{dpf}$ to cyanotoxic solutions as illustrated in Fig. 1. We did not observe any significant overt developmental phenotypes in any of the exposed larval zebrafish groups. We performed Kaplan-Meir survival analysis with a MantelCox Log-rank test to determine if BMAA, AEG, and 2,4DAB significantly alter survival over time. While there was a trend towards a slight decrease in viability, AEG did not significantly decrease larval survival when compared to the control group $(P=0.0531)$. Larval survival after exposure to either BMAA or 2,4-DAB was significantly reduced by $16 \%$
$(P=0.0008)$ and $50 \%(P<0.0001)$, respectively (Fig. 2A). Surprisingly, 2,4-DAB was the most potent cyanotoxin, and its EC50 for induced death is $500 \mu \mathrm{M}$. The survival model standard errors, coefficients, and probability of survival values are included in Table 1 . These data indicate that 2,4$\mathrm{DAB}$ is highly toxic to zebrafish larvae at moderately low concentrations.

When we assessed the survival of zebrafish larvae exposed to the binary and three-component mixtures of BMAA, AEG, and 2,4-DAB, we observed a trend towards increased mortality (i.e., ranging from 11.2 to $31.5 \%$ ) for the larval group exposed to the cyanotoxic mixtures containing increasing concentrations of 2,4-DAB (Fig. 2B). Thus, 2,4$\mathrm{DAB}$ alone drives the reduced viability of zebrafish larvae exposed to cyanotoxic mixtures, and when combining it with other cyanotoxin, toxicity is not enhanced. Then, we tested if there existed a significant relationship between 2,4-DAB and decreased viability using the simplex axial design. Figure $2 \mathrm{C}$ illustrates the viability across all mixture conditions, and it indicates a plot of viscosity as a function of all three-mixture components, BMAA, AEG, and 2,4-DAB. The grid lines representing percent viability decrease in value towards the 2,4-DAB vertex. At $500 \mu \mathrm{M}, 2,4-\mathrm{DAB}$ exhibited the lowest viability (Fig. $2 \mathrm{C}$ ). The analysis of variance indicates that a linear model is statistically significant $(P<0.0001)$ (Table 2).

Thus, we conclude that these three cyanotoxins do not interact to decrease survival in vivo and that 2,4-DAB is more toxic than BMAA and AEG, meaning the viability response tracks linearly with 2,4-DAB alone. The response is modeled by the below linear model in which $Y_{i}$ represents the predicted response, $\beta o$ is the intercept coefficient, and $\beta_{i}$ is the coefficient of the linear regression.

$Y i=\beta o+\sum_{i=1}^{n} \beta i X i$

\section{Mixture of Cyanotoxins Modulates Spontaneous Behavior in Larval Zebrafish}

All zebrafish larvae that survived to $6 \mathrm{dpf}$ had no overt developmental or morphological phenotypes. To determine if exposure to BMAA, AEG, and 2,4-DAB alone or in combination causes neurotoxicity, we first examined spontaneous locomotion across all 10 treatment groups (Fig. 3). At 6 $\mathrm{dpf}$, larvae were adapted to the testing conditions, and then their spontaneous movements were recorded for $18.5 \mathrm{~min}$ using a high-throughput behavior platform and automated FLOTE tracking software (Martin et al. 2020). We detected no significant differences in total distance traveled for larvae treated with BMAA, AEG, or 2,4-DAB compared to their respective vehicle controls (Fig. 3A). Thus, exposure 
Fig. 2 Viability assessment. A Kaplan-Meier cumulative survival plots comparing percent survival between four treatment groups: Control $(n=108)$, BMAA $(n=162)$, AEG $(n=162)$, and 2,4-DAB $(n=108)(P<0.001$, MantelCox log-rank test). B Bar graph representing percent viability across all treatment groups. $\mathbf{C}$ 2D contour plot representing zebrafish percent viability in response to each mixture of BMAA, AEG, and 2,4-DAB (Linear regression model, $P<0.0001)$

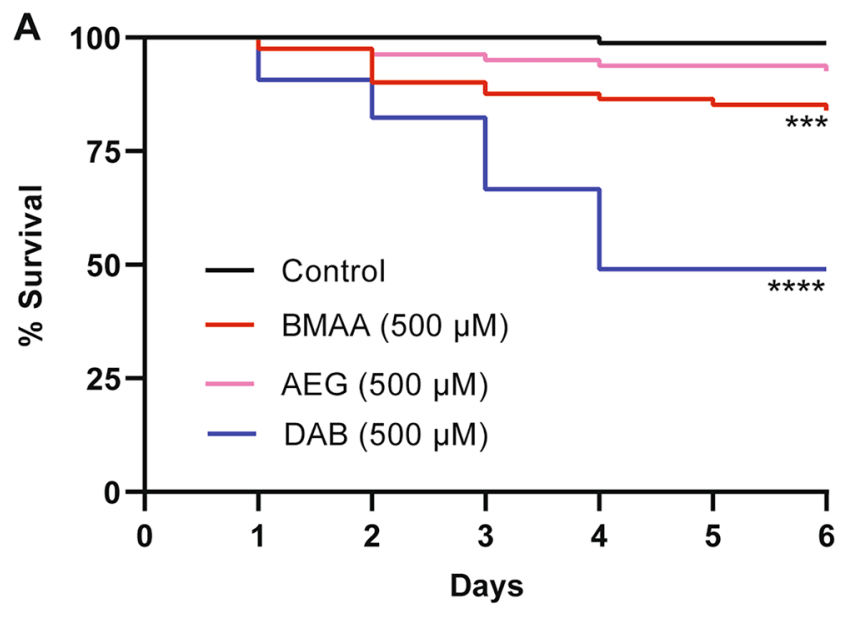

B

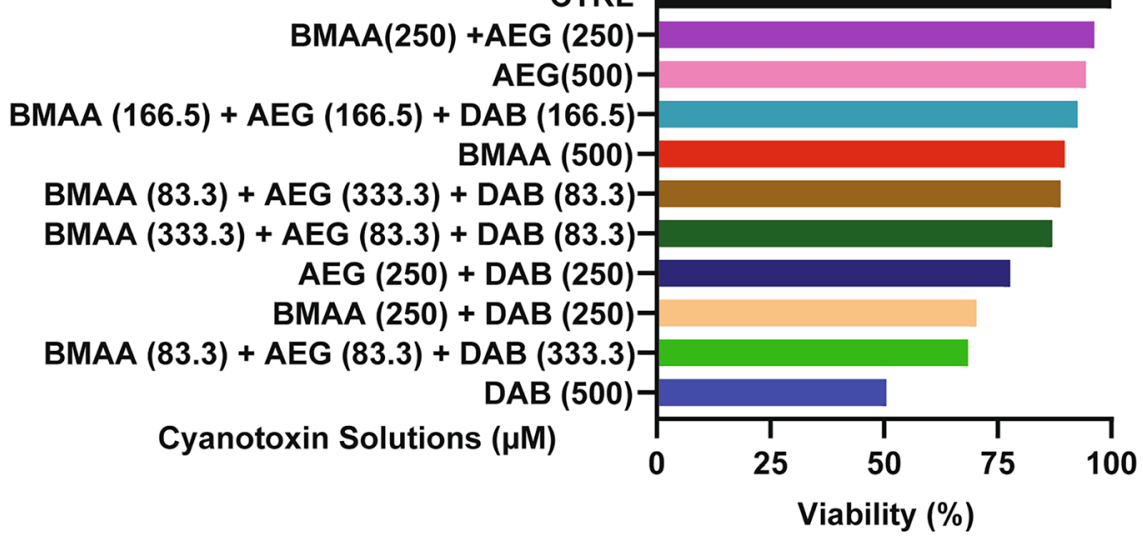

C

BMAA

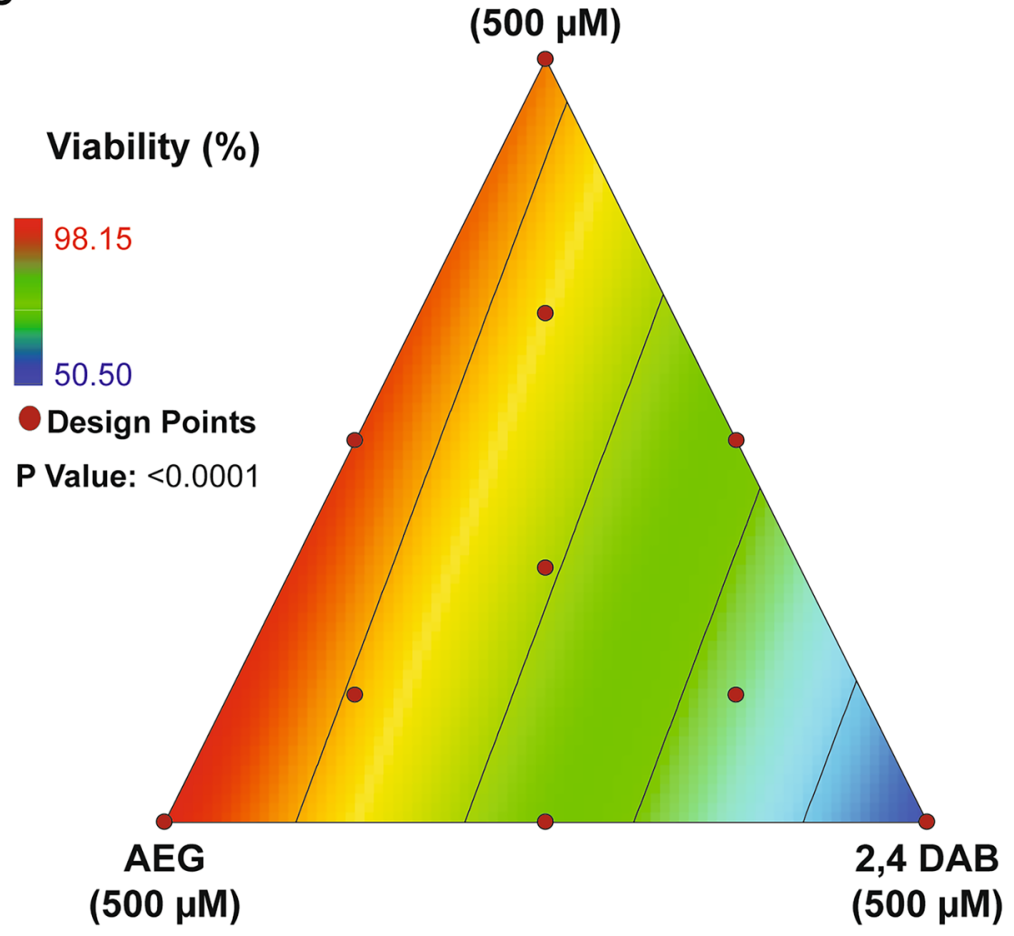


Table 1 Fitted survival probability and coefficient intervals

\begin{tabular}{lllll}
\hline Treatment & Std Error & Lower 95\% & Upper 95\% & Prob Survival \\
\hline Control & 0.01224 & 0.00173 & 0.08226 & 0.98768 \\
BMAA & 0.04095 & 0.09618 & 0.25833 & 0.83856 \\
AEG & 0.02914 & 0.03372 & 0.15543 & 0.92581 \\
2,4-DAB & 0.04682 & 0.41143 & 0.59294 & 0.49774 \\
\hline
\end{tabular}

to BMAA, AEG, or 2,4-DAB alone does not grossly impact motor function. Although the average speed of the BMAA and 2,4-DAB-exposed groups was not altered when compared to their respective controls, the average speed of the larvae treated with AEG was significantly reduced (Fig. 3A). The binary mixtures containing AEG also decreased spontaneous movement, with AEG plus BMAA reducing speed and AEG plus 2,4-DAB decreasing both distance traveled and speed (Fig. 3B). We also observed that exposure to the equimolar three-component mixture significantly decreased both distance traveled and average speed (Fig. 3C). DOE models were unable to fit these data at a statistically significant level, precluding any determination of whether there were interactions between toxins in this assay. These data indicate that although individual concentrations of BMAA and AEG do not substantially reduce survival of larval zebrafish, combined they can induce toxicity at a behavioral level.

\section{The Cyanotoxin 2,4-DAB Does Not Alter Acoustic Startle Sensitivity, but BMAA and AEG Do}

To determine if exposure to BMAA, AEG, and 2,4-DAB alone or in combination affects specific sensorimotor behaviors in larval zebrafish, we measured acoustic startle frequency in response to a range of stimulus intensities in the same 10 groups described above. The frequency of Mauthner cell-dependent short-latency C-bends (SLCs) is displayed in Fig. 4A-C. We previously found that BMAA increases SLC frequency, suggesting increased excitability of the startle circuit (Martin et al. 2020). Here, we found that like BMAA, AEG also increased SLC frequency, but 2,4-DAB did not (Fig. 4A). We also observed that the binary mixture of BMAA and AEG caused a similar increase in SLC frequency compared to BMAA and AEG alone (Fig. 4B). No other binary or three-component mixture induced statistically significant effects compared to controls. We also analyzed the frequency of long latency C-bends (LLCs), which are normal responses typically elicited by weaker stimuli and that are independent of the Mauthner cell and instead rely on the activation of prepontine neurons (Marquart et al. 2019). Neither BMAA nor its isomers significantly altered LLC response frequency (Supplemental Fig. 2). These data indicate that BMAA and AEG specifically enhance the sensitivity of the SLC circuit. We next modeled the SLC experiment by fitting the same linear equation described above. Figure 4D shows the grid lines representing the SLC response across all 10 tested mixtures. SLC sensitivity increased towards the pure doses of BMAA $(500 \mu \mathrm{M})$ and AEG $(500 \mu \mathrm{M})$ as well as their binary mixture (BMAA at $500 \mu \mathrm{M}$ plus AEG at $500 \mu \mathrm{M})$. The analysis of variance indicates that the linear model is statistically significant $(P<0.0001)$ (Table 3$)$. These data reveal that BMAA and AEG alone can augment the SLC response and that they interact, likely additively, at lower concentrations to significantly enhance SLC responses in larval zebrafish. These data also reveal that 2,4-DAB alone or in combination with BMAA and/or AEG does not affect the initiation of the SLC response.

\section{The Cyanotoxin 2,4-DAB Modulates Acoustic Startle Kinematics in Larval Zebrafish}

To assess whether cyanotoxin mixtures cause more subtle defects in motor control, we examined the kinematic performance of SLC responses in the exposed larvae, as these rapid movements require coordinated activation of large sets of motor neurons and muscle. Using FLOTE tracking software, we looked at a set of kinematic parameters including latency, body curvature, angular velocity, and duration (Burgess and Granato 2007a, b). We found that 2,4-DAB at $500 \mu \mathrm{M}$, but not BMAA or AEG, increased the curvature of the $\mathrm{C}$-bend response, as shown in three representative examples (Fig. 5A) and quantified in Fig. 5C. We also observed that the average latency of response in fish exposed to BMAA, and AEG was significantly reduced (Fig. 5B). The BMAA/AEG binary mixture produced the same effect on latency as the individual toxins (Supplemental Fig. 3A), indicating that at $250 \mu \mathrm{M}$ BMAA and
Table 2 Analysis of variance (ANOVA): viability

\begin{tabular}{lclccc}
\hline & Sum of Squares & DF & Mean Square & F Value & P Value \\
\hline Model & 3205.63 & 2 & 1602.82 & 68.01 & $<0.0001$ \\
Linear mixture & 3205.63 & 2 & 1602.82 & 68.01 & $<0.0001$ \\
Residual & 259.24 & 11 & 23.57 & & \\
Lack of fit & 202.66 & 7 & 28.95 & 2.05 & 0.2549 \\
Pure error & 56.58 & 4 & 14.15 & & \\
\hline
\end{tabular}

ANOVA results, showing the significance of a linear model ( $D F$, degrees of freedom) 
A
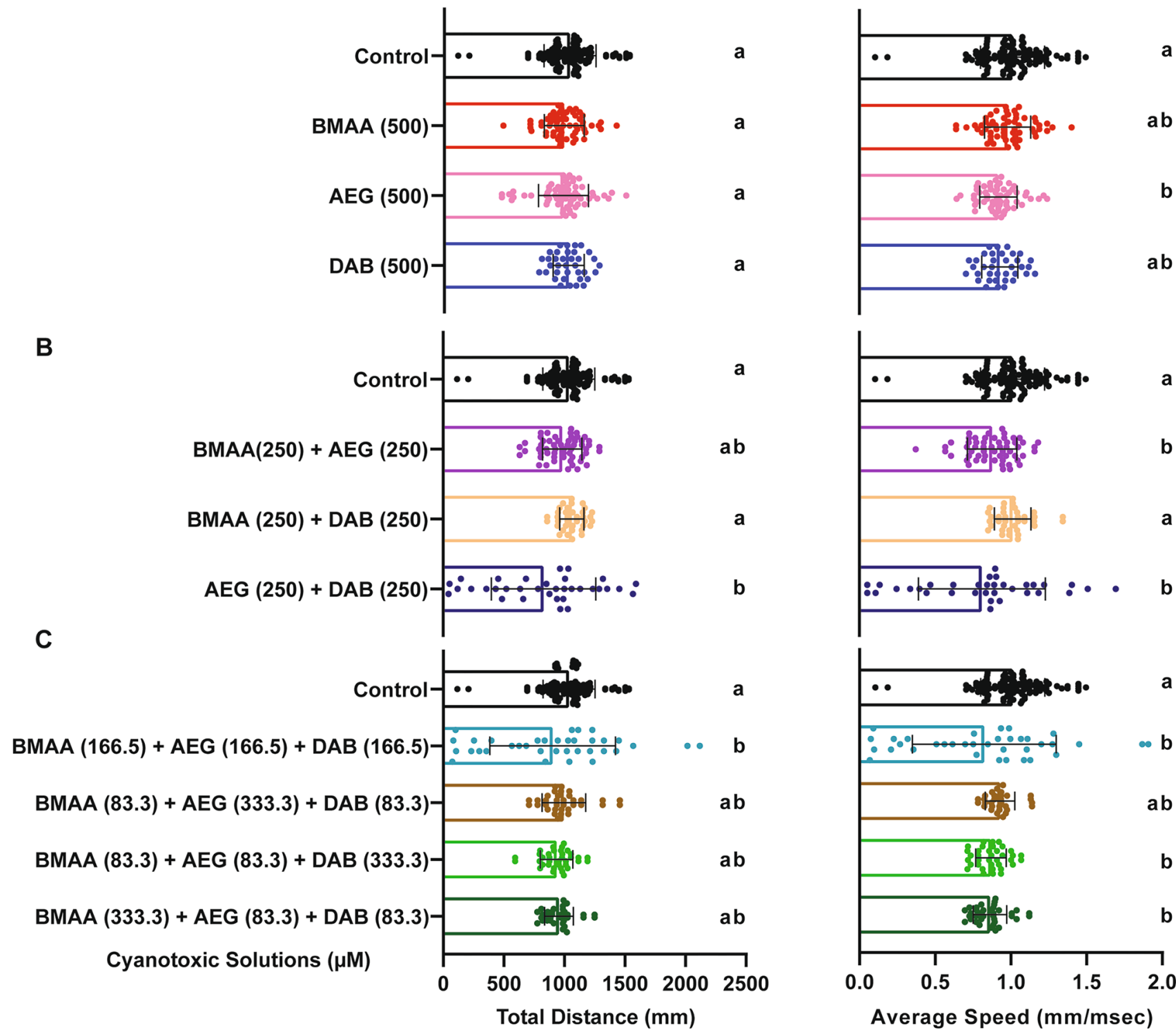

a

$\mathbf{a b}$

b

ab
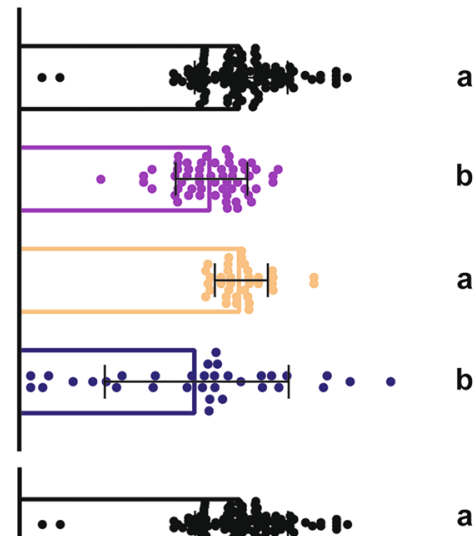

a
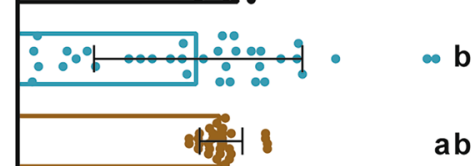

$\mathbf{a b}$

b

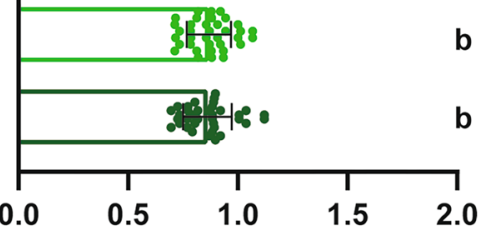

Average Speed $(\mathrm{mm} / \mathrm{msec})$

Fig. 3 Spontaneous Locomotion Response. A-C Bar graphs represent the total distance traveled during the $18.5 \mathrm{~min}$ spontaneous movement assay for each larva and average speed across the same assay for BMAA, AEG, and 2,4-DAB alone, their binary mixtures, and three-component mixtures, respectively. Levels not connected by the same letter are significantly different (Tukey-Kramer HSD, Alpha $0.05)$

the increase in curvature. Consistent with the increase in curvature we observed, the 2,4-DAB-exposed larvae also displayed higher angular velocity and longer duration of the SLC response (Fig. 5F, G). Together, our behavioral analyses show that AEG affects larval zebrafish behavior similarly to BMAA, while 2,4-DAB alters distinct aspects of behavior from the other two toxins, playing a critical role in regulating coordinated movements in the zebrafish larvae model. 
Fig. 4 BMAA and AEG augment the short-latency c startle (SLC) response. A-C Bar graphs display the distribution of the short-latency C-bend (SLC) sensitivity indices for each tested larva after exposure to individual cyanotoxins, binary mixtures, and threecomponent mixtures, respectively. SLC sensitivity index is determined for each fish by calculating the area under the curve of SLC frequency vs. stimulus intensity $(n=108$ siblings; mean \pm SEM). Levels not connected by the same letter are significantly different (TukeyKramer HSD, Alpha 0.05). D 2D contour plot for the SLC sensitivity at different points in the design space, including each individual cyanotoxin (BMAA, AEG, and 2,4-DAB) and their seven different mixture ratios (Linear regression model, $P=0.0257$ )
A

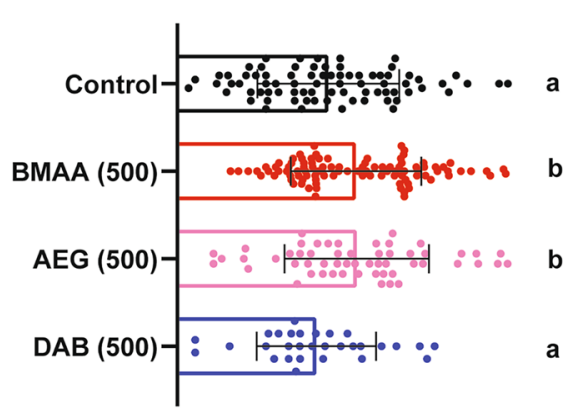

B

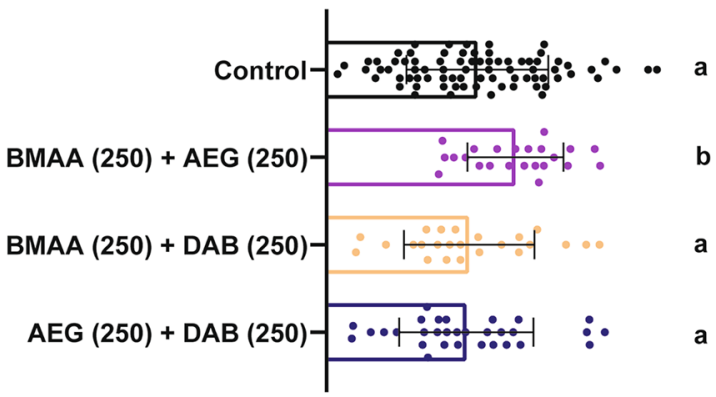

C

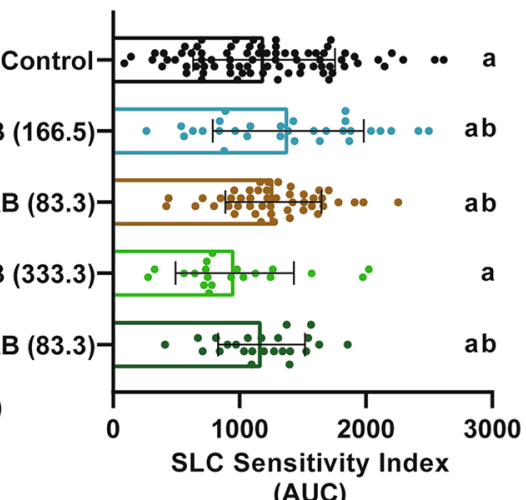

(AUC)

D

Startle Sensitivity Index (AU)

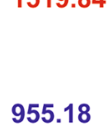

Design Points

P Value: 0.0257

BMAA

$(500 \mu \mathrm{M})$

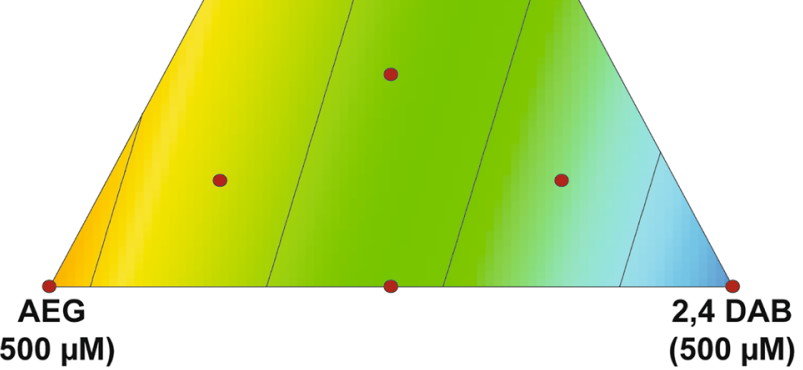


Table 3 Analysis of variance (ANOVA): short-latency C startle

\begin{tabular}{llllll}
\hline & $\begin{array}{l}\text { Sum of } \\
\text { Squares }\end{array}$ & DF & $\begin{array}{l}\text { Mean } \\
\text { Square }\end{array}$ & F Value & P Value \\
\hline Model & $2.148 \mathrm{E}+005$ & 2 & $1.074 \mathrm{E}+005$ & 5.20 & 0.0257 \\
$\begin{array}{l}\text { Linear mix- } \\
\quad 2.148 \mathrm{E}+005\end{array}$ & 2 & $1.074 \mathrm{E}+005$ & 5.20 & 0.0257 \\
$\quad$ Residual & $2.271 \mathrm{E}+005$ & 11 & $20,641.63$ & & \\
Lack of fit & $1.253 \mathrm{E}+005$ & 7 & $17,906.09$ & 0.70 & 0.6790 \\
Pure error & $1.017 \mathrm{E}+005$ & 4 & $25,428.83$ & & \\
\hline
\end{tabular}

ANOVA results, showing the significance of a linear model $(D F$, degrees of freedom)

\section{Exposure to 2,4-DAB Affects Biological Processes and Protein Homeostasis In Vivo}

Our data show that 2,4-DAB not only alters motor function during the startle response but more significantly reduces viability of larval zebrafish by approximately $50 \%$ by 4 days of development (Fig. 2A). To understand the molecular processes by which 2,4-DAB may exert these effects we used a "bottom-up" shotgun proteomics approach. To identify both the mechanisms that may cause death as well as those that may induce behavioral neurotoxicity, we collected larval zebrafish exposed to $500 \mu \mathrm{M}$ 2,4-DAB at two time points: 2 dpf, prior to most of the toxin-induced mortality, and $4 \mathrm{dpf}$, after the wave of mortality had passed (Fig. 2A). Control and 2,4-DAB-exposed larvae were each pooled in three biological replicates and flash frozen in liquid nitrogen, followed by proteomic analysis via LC-MS/MS. Approximately 2200 proteins were identified in each sample.

First, we analyzed the regulation of protein abundance by identifying all differentially expressed proteins (DEPs) in the 2,4-DAB samples by comparing the mean abundance at each time point (i.e., $2 \mathrm{dpf}$ and $4 \mathrm{dpf}$ ) to their respective controls using a two-way Student's $t$-test $(P<0.05)$. All DEPs in both larval groups along with their significance values and fold change can be found in Supplemental Table 1, and volcano plots illustrating the direction of protein regulation at both time points are shown in Supplemental Fig. 4. Overall, changes in protein levels at $2 \mathrm{dpf}$ were less pronounced than those at $4 \mathrm{dpf}$. A total of 102 and 398 differentiated expressed proteins (DEPs) were identified for the $2 \mathrm{dpf}$ and $4 \mathrm{dpf}$ groups, respectively, representing a fourfold increase in the number of dysregulated proteins in the $4 \mathrm{dpf}$ samples (Fig. 6A). There were no DEPs that were dysregulated at both time points, as illustrated by the absence of overlap in the Venn diagram in Fig. 6A. These results suggest that 2,4-DAB affects an entirely different set of molecules during early embryonic development than at later larval stages.

The top 10 up and downregulated proteins in the $4 \mathrm{dpf}$ group (Fig. 6B) included several associated with apoptosis (API5, PEA15) and metabolic function (MPC1, FTCD,
Fig. 5 2,4-DAB modulates kinematics of the $\mathrm{C}$ startle response. A Three representative images of the peak SLC curvature in 6 days post fertilization (dpf) larvae exposed to vehicle (control, top) and to 2,4-DAB at $500 \mu \mathrm{M}$ (bottom). B and $\mathbf{C}$ Bar graphs quantifying the responses latency and curvature, respectively. $\mathbf{D}$ and $\mathbf{E} 2 \mathrm{D}$ contour plot for both the latency and curvature responses at different points in the design space, including each individual cyanotoxin (BMAA, $\mathrm{AEG}$, and 2,4-DAB) and their seven different mixture ratios (Linear regression model, $P<0.05$ ). $\mathbf{F}$ and $\mathbf{G}$ Bar graphs quantify responses maximum angular velocity and duration, respectively. Levels not connected by the same letter are significantly different (Tukey-Kramer HSD, Alpha 0.05)

UOX). To determine whether these functions were significantly affected by $2,4-\mathrm{DAB}$ at both time points, we performed gene ontology (GO) enrichment analysis to reveal the classification of the biological processes represented by all DEPs. DEPs were classified into three major functional groups, including metabolism, development, and cellular functions. As shown in Fig. 6C, both time points reveal the dysregulated proteins are largely involved in metabolic processes ( $2 \mathrm{dpf}: 56 \%$; $4 \mathrm{dpf}: 48 \%$ ), cellular processes ( $2 \mathrm{dpf}$ : 23\%; 4 dpf: $29 \%$ ), and developmental processes (2 dpf: $21 \%$; $4 \mathrm{dpf}: 7 \%$ ). A list of all subgroups of impacted biological processes can be found in Supplemental Tables 3 and 4 . We next used Ingenuity Pathway Analysis (IPA) software to identify perturbed canonical pathways following exposure to 2,4-DAB. Canonical pathways were predicted using a Fisher's exact $t$-test to determine the probability that DEPs from the dataset correspond with targets which are known to be activated/inhibited by those molecules based on knowledge in the Ingenuity database (Krämer et al. 2014). Only two canonical pathways were significantly enriched at 2 dpf: (1) neuronal nitrous oxide synthetase (nNOS) signaling and (2) protein ubiquitination pathway (Supplemental Fig. 5A). Moreover, only eight proteins were significantly dysregulated within these canonical pathways in our samples (Supplemental Fig. 5B), but both canonical pathways are associated with neuropathology (Atkin and Paulson 2014; Yi et al. 2009). We identified 56 canonical pathways that were significantly impacted in the $4 \mathrm{dpf}$ samples (Supplemental Table 2). Figure 6D shows the top 20 canonical pathways that were predicted to be altered by $2,4-\mathrm{DAB}(500 \mu \mathrm{M})$ exposure, based on a $p$-value less than 0.05 and an activation $z$-score that was greater than an absolute value of 2 . In agreement with our GO analysis, several of these canonical pathways are involved in cellular processes such as energy metabolism and protein homeostasis. For instance, both gluconeogenesis and glycolysis pathways were predicted to be inhibited $(z$-score $<-2)$, leading to the predicted activation of metabolic diseases such as glucose metabolism disorders $(z$-score $=2.227$; overlap $p$-value $=1.46 \mathrm{E}-03)$. Moreover, five key regulators in the data set (HSPA5, CAT, SOD2, HSP90, and SOD1) were altered in the direction consistent with increased protein damage $(z$-score $=2.219$; overlap 
A

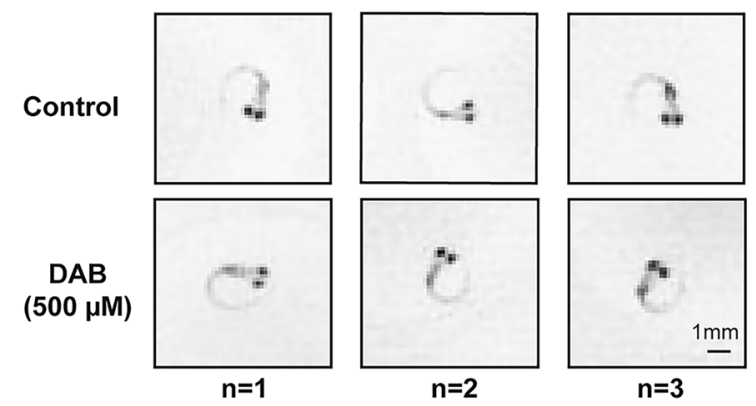

B

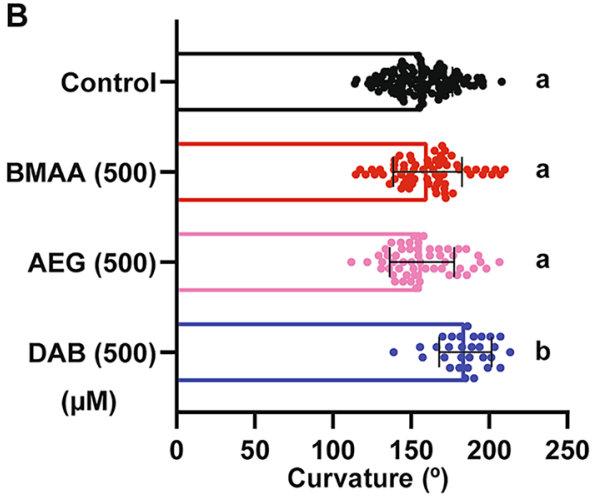

D

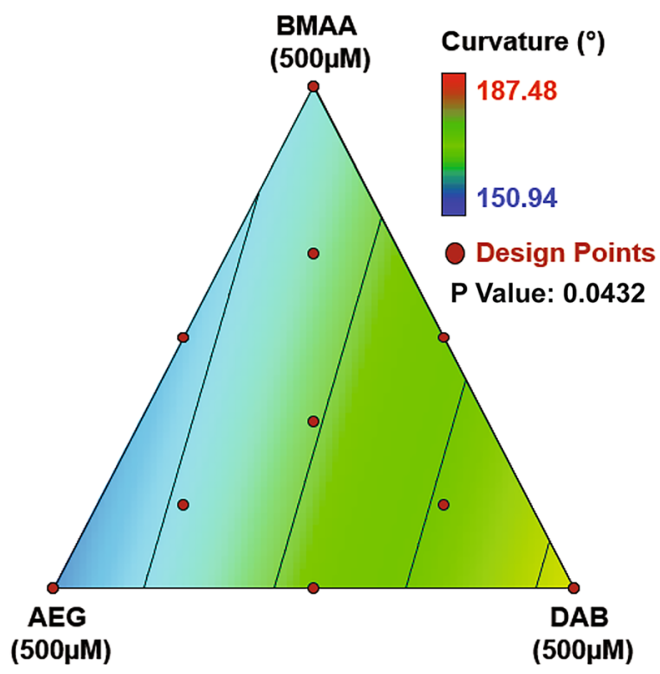

F

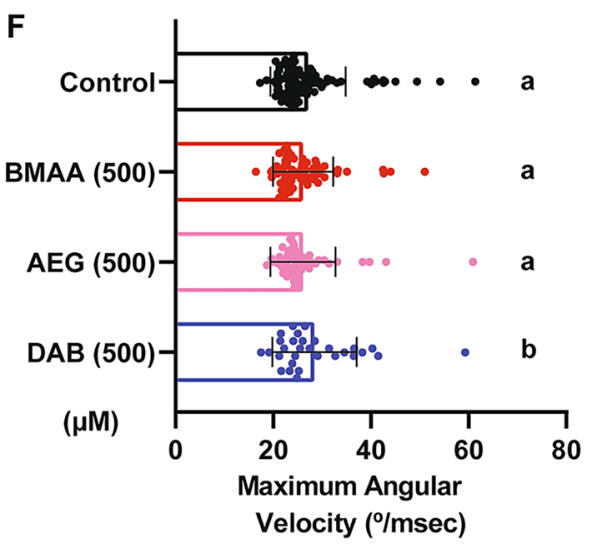

C

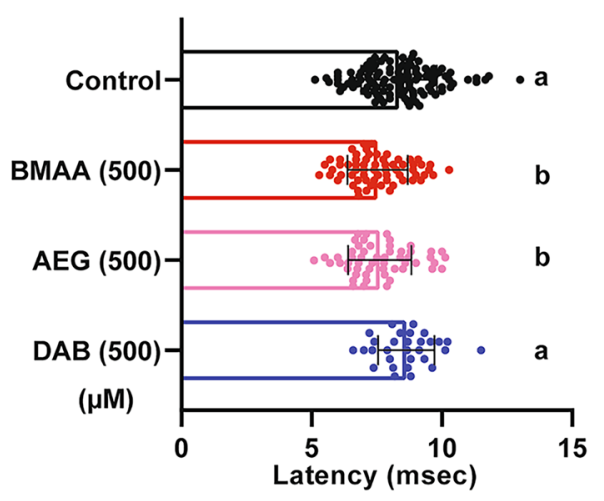

E

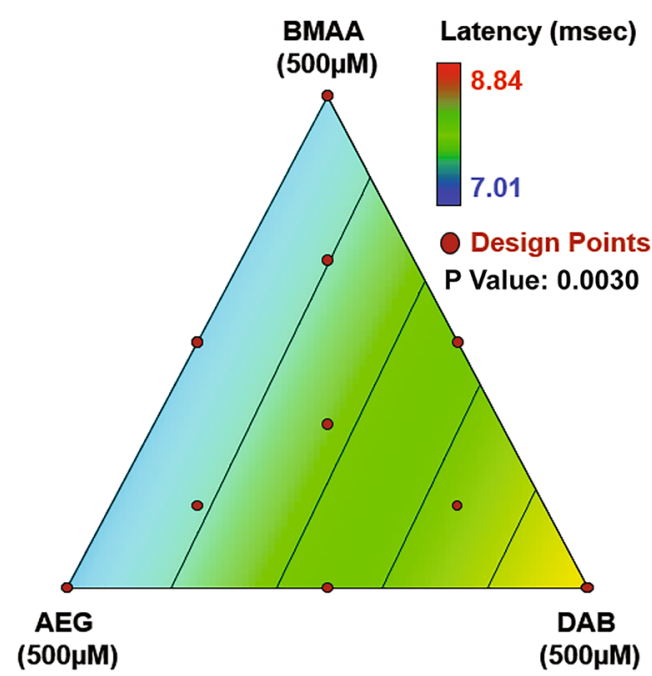

G

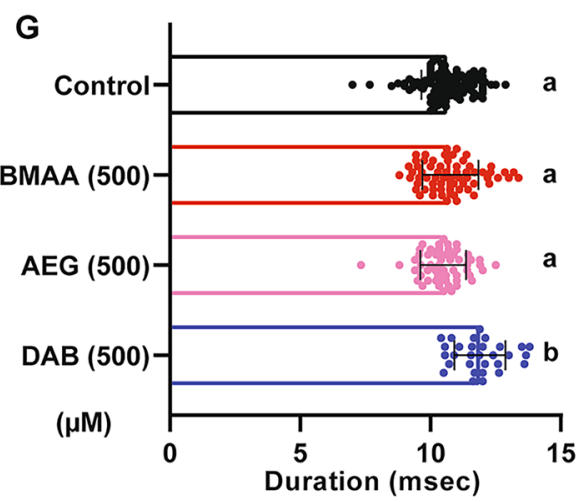


Table 4 Analysis of variance (ANOVA): latency

\begin{tabular}{llllll}
\hline & $\begin{array}{l}\text { Sum of } \\
\text { squares }\end{array}$ & DF & $\begin{array}{l}\text { Mean } \\
\text { square }\end{array}$ & $\boldsymbol{F}$ value & $\boldsymbol{P}$ value \\
\hline Model & 1.65 & 2 & 0.83 & 10.27 & 0.0030 \\
$\begin{array}{l}\text { Linear mix- } \\
\text { ture }\end{array}$ & 1.65 & 2 & 0.83 & 10.27 & 0.0030 \\
Residual & 0.89 & 11 & 0.081 & & \\
Lack of fit & 0.56 & 7 & 0.080 & 0.97 & 0.5453 \\
Pure error & 0.33 & 4 & 0.082 & & \\
\hline
\end{tabular}

ANOVA results, showing the significance a linear regression model ( $D F$, degrees of freedom)

$p$-value $=2.67 \mathrm{E}-04)$. These proteins are known to decrease damage of proteins in neurons (Mattson 2006; Murakami et al. 2011), and they were all found to be downregulated in our samples.

During normal development from 2 to $4 \mathrm{dpf}$, widespread changes in gene expression occur. To determine how 2,4DAB exposure affects this developmental program, we identified DEPs between the 2 and 4 dpf control group and compared this set of proteins to the DEPs that were found between the 2 and $4 \mathrm{dpf}$ 2,4-DAB exposed group. This systematic analysis revealed 868 DEPs for the control group and 606 DEPs for the 2,4-DAB-exposed group, with 349 DEPs common to both groups (Fig. 7A). We found that DEPs in the control group were mostly upregulated while those in the 2,4-DAB-exposed group were mostly downregulated, perhaps reflecting a widespread impairment in the ability to activate gene expression (Supplemental Fig. 6). Our enrichment analysis of control and 2,4-DAB DEPs revealed 7 highly enriched canonical pathways (overlap $p$-value $<0.05$ and $z$-score $>|2|$ ) (Fig. 7B). In agreement with our previous enrichment analysis showing downregulation at $4 \mathrm{dpf}$ (Fig. 6C), glycolysis and gluconeogenesis were both strongly activated in the control samples but only mildly activated in the 2,4-DAB samples. Protein kinase A and 14-3-3 protein signaling were similarly impaired in their activation by $2,4-\mathrm{DAB}$ relative to

Table 5 Analysis of variance (ANOVA): curvature

\begin{tabular}{llllll}
\hline & $\begin{array}{l}\text { Sum of } \\
\text { squares }\end{array}$ & DF & $\begin{array}{l}\text { Mean } \\
\text { square }\end{array}$ & $\boldsymbol{F}$ value & $\boldsymbol{P}$ value \\
\hline Model & 578.8 & 2 & 289.4 & 4.2 & 0.0432 \\
$\begin{array}{l}\text { Linear mix- } \\
\quad 578.8\end{array}$ & 2 & 289.4 & 4.2 & 0.0432 \\
$\quad$ Residual & 750.9 & 11 & 68.3 & & \\
Lack of fit & 683.3 & 7 & 97.6 & 5.8 & 0.0548 \\
Pure error & 67.7 & 4 & 16.9 & & \\
\hline
\end{tabular}

ANOVA results, showing the significance a linear regression model (DF, degrees of freedom) control, while calcium signaling was more strongly activated by $2,4-\mathrm{DAB}$ than in controls.

To further investigate how 2,4-DAB impacts developmental protein regulation, we next performed upstream regulation and disease prediction analyses of the control and 2,4-DAB DEPs. The upstream regulator analysis evaluates linkage to DEPs through coordinated expression. Thus, it identifies potential upstream regulators (i.e., transcription factors or signaling proteins) that have been observed experimentally to affect protein expression (Krämer et al. 2014). Here, we have presented a subset of the top-scored (overlap $p$-value $<0.05$ and $z$-score $>|2|$ ) upstream regulators and diseases (Fig. 7C, D). All activated and inhibited upstream regulators can be found in Supplemental Table 7. Among the top upstream regulators, we found that RICTOR was aberrantly strongly activated in the 2,4-DAB samples ( $z$-score $=5.18$; Fig. 7C) but not the control samples. RICTOR is a key component of mTOR signaling, which was highly enriched in our samples ( $p$-value $=0.0007$; Supplemental Table 6), and regulates cell proliferation and neurodevelopment (Karalis and Bateup 2021). Two additional upstream regulators and known transcription factors, NFE2L1 and 2, were predicted to be inhibited (overlap $z$-score $=-2.83$ ) in the 2,4-DAB exposed samples (Fig. 7C). NFE2L1 and NFE2L2 are involved in regulation of several downstream molecules involved in protective responses due to injury and inflammation (Saha et al. 2020). In addition to these regulators, multiple other neurodegeneration-related factors were identified (Fig. 7C; Supplemental Table 6). Several diseases and biofunctions that implicate motor control were affected by the changes in protein expression from 2 to $4 \mathrm{dpf}$ in control and 2,4-DAB-exposed fish (Fig. 7D). Control samples showed an increase in muscle contractility and neurotransmitter secretion that was absent from the 2,4-DAB samples, and controls had decreased association with ataxia and movement disorders that were not seen in 2,4-DAB. These changes suggest reduced motor function in the 2,4-DAB-exposed larvae, which is consistent with the altered startle kinematics we observed in our behavioral analysis (Fig. 5).

Finally, we explored the effects of 2,4-DAB on biological networks in zebrafish larvae. We found significant differential regulation of key proteins associated with cellular damage and inflammatory response (Supplemental Table 5). Within the highest scored network (score $=68$ ), we found that the ALS-associated superoxide dismutase 1 (SOD1; Enrichment $p$-value $=0.012 ; \log _{2}$ fold-change $=-1.41$ ) was significantly downregulated in our samples. We also found that another ALS-associated protein, ubiquilin 4 (Brettschneider et al. 2012; Edens et al. 2017), was one of the top 10 most downregulated proteins in the $4 \mathrm{dpf} 2,4-$ DAB samples (UBQLN4; Enrichment $p$-value $=0.012 ; \log _{2}$ fold-change $=-3.67$; Fig. $6 \mathrm{~B}$ ). These data demonstrate the 
Fig. 6 A Venn diagram shows no overlap in the differentially Expressed Proteins (DEPs) between 2,4-DAB $(500 \mu \mathrm{M})$ zebrafish larvae exposed groups at $2 \mathrm{dpf}$ (left) and $4 \mathrm{dpf}$ (right). B Top 10 DEPs found to be up or downregulated in the zebrafish larvae groups that were exposed to 2,4-DAB from $6 \mathrm{hpf}$ until 4 days post fertilization (4 dpf). C Pie chart showing the percentage of enriched GO Biological Processes derived from each set DEPs time points: 2- and 4-day post fertilization. D Top 20 canonical pathways found to be activated or inhibited in the zebrafish larvae groups that were exposed to 2,4-DAB until 4 days post fertilization ( $4 \mathrm{dpf}$ ). Red shading indicates predicted activation $(z$-score $>2)$, and blue shading indicates predicted inhibition $(z$-score $<-2)$. This bar graph width represents enrichment values in the form of minus $\log$ of $p$ value that were greater than 1.3
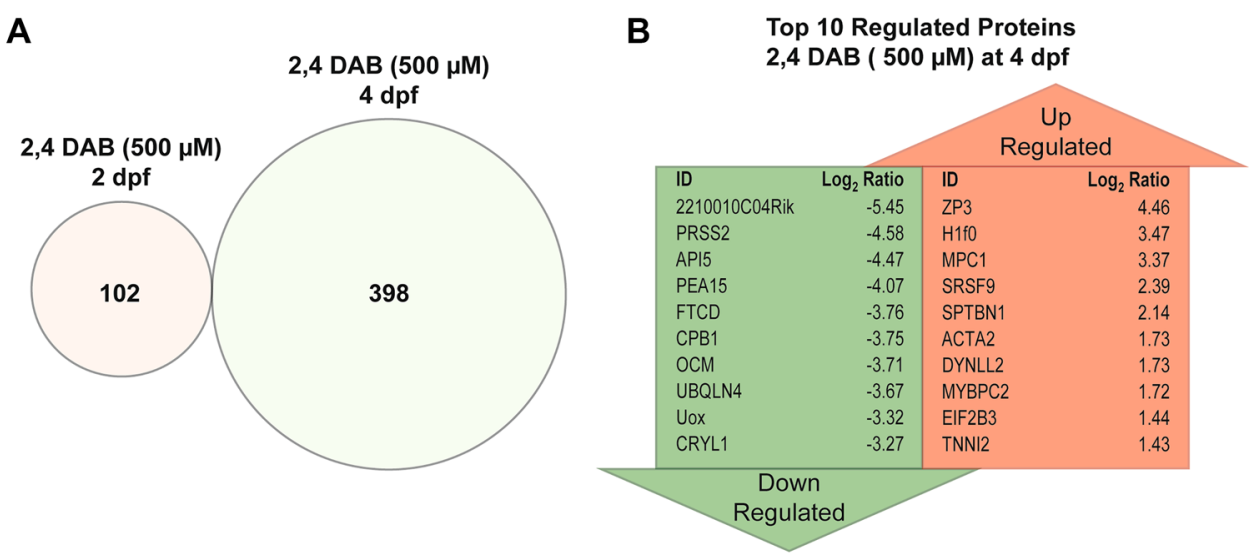

C
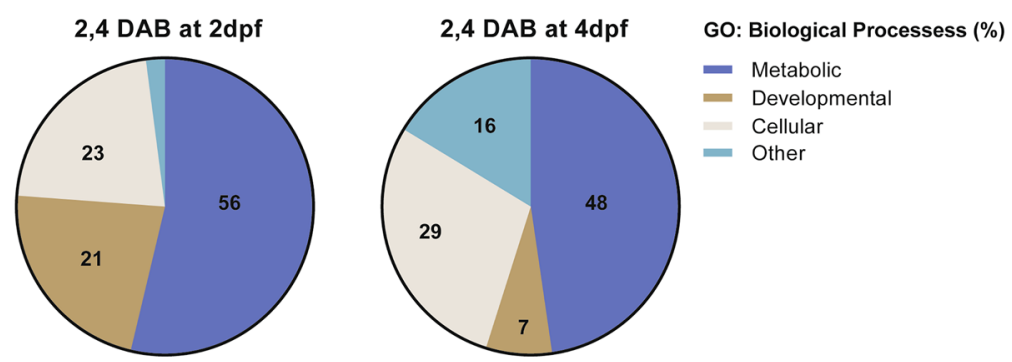

D

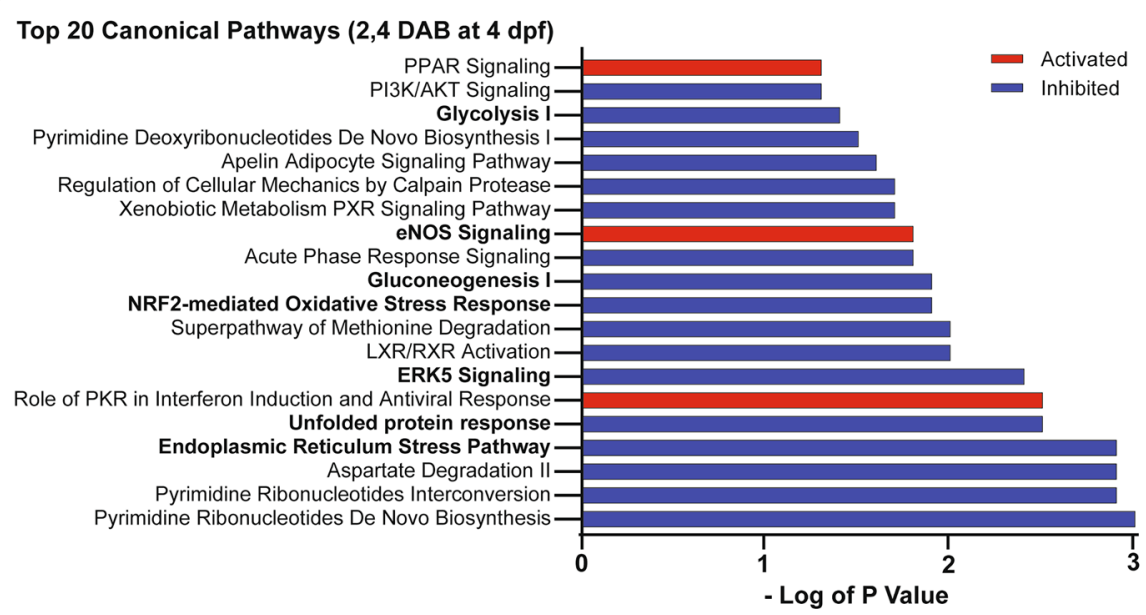

power of proteomic screens to identify developmental effects of toxin exposures. Furthermore, this study indicates that 2,4-DAB impacts neurodegenerative pathways in larval zebrafish, highlighting the importance of further exploring the links between BMAA isomers and neural disease.

\section{Discussion}

Here, we provide a detailed report of the in vivo toxic effects of the beta-n-methylamino-1-alanine (BMAA) isomer 2,4-diaminobutyric acid (2,4-DAB) on zebrafish larvae. For many years, BMAA has been studied for its association with sporadic neurodegenerative disease, with several epidemiological studies revealing clusters of cases linked with environmental exposure to BMAA (Caller et al. 2009; Field et al. 2013; Masseret et al. 2013). However, BMAA is produced by cyanobacteria alongside its structural isomers 2,4DAB and N-(2-aminoethyl) glycine (AEG) in natural algal blooms (Jungblut et al. 2018; Lance et al. 2018; Vo Duy et al. 2019). Although BMAA is a well-established neurotoxin, the toxicity of its isomers is less clear. Some recent in vitro studies have found that AEG and 2,4-DAB are toxic to neurons in culture (Main et al. 2018; Martin et al. 2019), but whether they exert neurotoxic effects in vivo has not been established. To the best of our knowledge, only one 
A

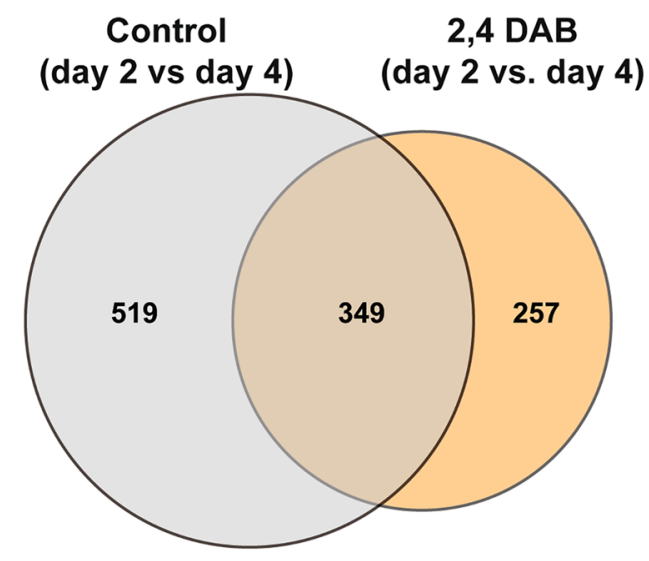

C

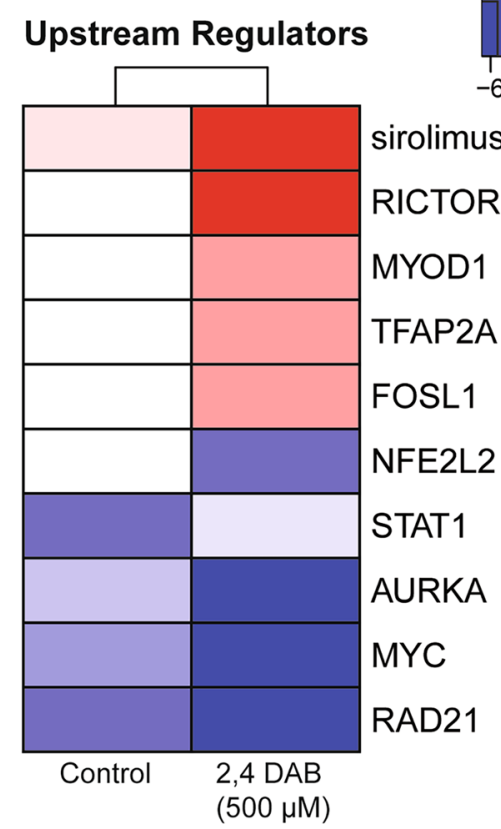

B

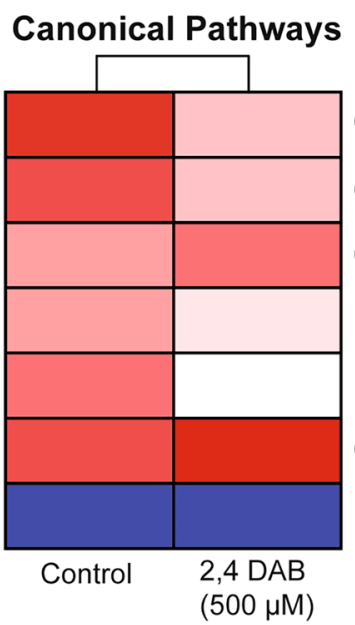

D

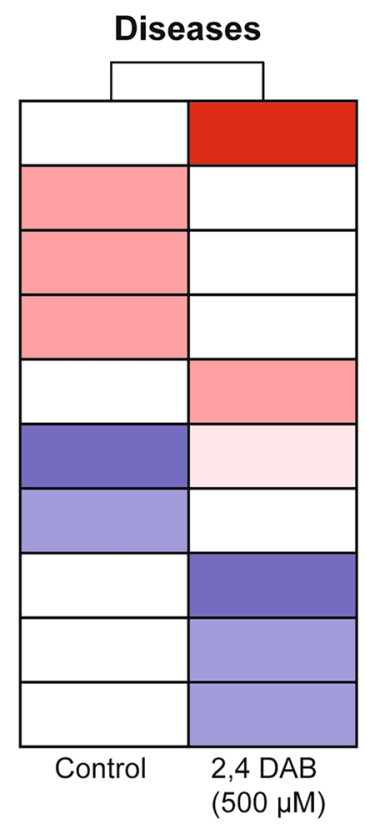

Glycolysis I

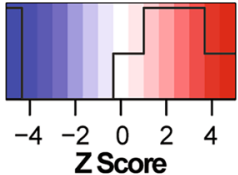

Gluconeogenesis I

Calcium Signaling

Protein Kinase A Signaling

14-3-3-mediated Signaling

Coronavirus Pathogenesis Pathway

EIF2 Signaling

Cell death

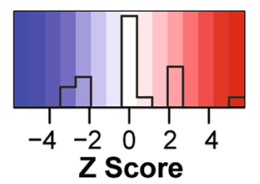

Induction of cells

Contractility of skeletal muscle

Secretion of neurotransmitter

Damage of protein

Ataxia

Movement Disorders

Cell viability

Autophagy

Modification of hydrogen peroxide
Fig. 7 Systematic proteomics analysis of developmental changes in protein expression in control and 2,4-DAB $(500 \mu \mathrm{M})$ treated zebrafish larvae. A DEPs were identified in by comparing $4 \mathrm{dpf}$ and $2 \mathrm{dpf}$ samples in control and 2,4-DAB-treated larvae. Venn diagram showing DEP overlap between control and the 2,4-DAB treatment. B Canonical pathways impacted during development from 2 to $4 \mathrm{dpf}$ in con-

prior study has tested the toxic effects of 2,4-DAB in animals (Ferraiuolo et al. 2019), while none have evaluated the toxicity of AEG in an animal model or examined whether these cyanotoxins interact. Thus, we aimed to measure both the individual and the combined toxic effects of BMAA, AEG, and 2,4-DAB in vivo. Using the larval zebrafish model, we identified impacts on organismal viability, sensorimotor behavior, and global protein expression after developmental exposure to 2,4-DAB. trol and 2,4-DAB samples. C Predicted upstream regulators. D Diseases and biological functions. The red- or blue-colored rectangles in each column indicate the $z$-score activities for each analysis. Red shading indicates predicted activation and blue shading indicates predicted inhibition. This heat map represents the activated of inhibited $z$-scores that were greater than the absolute value of 2

Our data show that 2,4-DAB is a more potent toxin than BMAA and AEG in vivo. The principle finding from our study is that $2,4-\mathrm{DAB}$ caused $50 \%$ mortality at $500 \mu \mathrm{M}$ after just 4 days of exposure, while BMAA and AEG caused very little mortality under the same conditions (Fig. 2). The effects of 2,4-DAB on viability are dose dependent, as the lowest observed effect level was $250 \mu \mathrm{M}$ (Supplemental Fig. 7). These results are supported by a previous dose response study using zebrafish larvae in which $300 \mu \mathrm{M}$ was sufficient 
to produce signs of cardiotoxicity (Ferraiuolo et al. 2019). These results contrast with those from in vitro studies in which 2,4-DAB did not elicit an effect on viability at concentrations lower than $2000 \mu \mathrm{M}$ or $1000 \mu \mathrm{M}$ in human liver/brain cancer cells or mouse motoneuron-like cells, respectively (Ferraiuolo et al. 2019; Martin et al. 2019). This distinction underscores the importance of studying toxin exposures in an in vivo context. Although exposure to BMAA or 2,4-DAB alone has previously been shown to cause morphological defects in larval zebrafish (Purdie et al. 2009; Ferraiuolo et al. 2019), we did not observe any significant morphological defects upon exposure to BMAA, AEG, or 2,4-DAB at $500 \mu \mathrm{M}$. This could be due to differences in zebrafish strain, embryo medium, exposure route, or observer analysis. However, the lack of overt defects in the present study is consistent with our previous report on BMAA and Microcystin LR (MCLR) exposure (Martin et al. 2020).

Real-world cyanotoxin exposures are likely to involve more than one toxin, and our previous studies indicate that BMAA, AEG, and 2,4-DAB can interact in vitro (Martin et al. 2019) and that BMAA and MCLR can interact in vivo (Martin et al. 2021). There are currently no guidelines regarding safe levels of these toxins, and although only traces of them are usually found in surface waters (Violi et al. 2019; Vo Duy et al. 2019), these cyanotoxins have been shown to bioaccumulate through the food web. For example, BMAA and 2,4-DAB have been found at relatively high concentrations $\left(\sim 0.02\right.$ to $\left.8 \mathrm{mg} \mathrm{kg}^{-1}\right)$ in freshwater fish, crustaceans, and other types of seafood as well as in the crop plant alfalfa (Medicago sativa) (Lance et al. 2018; Samardzic et al. 2021). Thus, our mixture paradigm is an approximate model of natural exposures. Building on these findings, here, we employed a simplex axial design to investigate the combined effects of exposure to BMAA, AEG, and 2,4-DAB in vivo. Although Design of Experiments (DOE) approaches are typically used to determine the optimum combination of constituents that generate an outcome (e.g., for drug formulations) (Politis et al. 2017), several studies have shown that DOE is also an excellent tool for toxicological investigations of mixtures (Groten et al. 2001; Pomati et al. 2008). Based on our results using the simplex axial design, we suggest evaluating multiple endpoints (e.g., viability, spontaneous movement, and startle kinematics) to further establish the significance of observed effects. Even though BMAA, AEG, and 2,4-DAB did not cause substantial effects on spontaneous locomotion on their own, exposure to the binary mixtures resulted in a significant modulation, decreasing both total distance traveled and average speed (Fig. 3). Our spontaneous movement data also show that while AEG only exerts a minor effect on viability of larval zebrafish, it still can induce neurotoxicity at a behavioral level, and that effect was enhanced when AEG was combined with 2,4-DAB (Fig. 3).

Our analysis of sensorimotor function using an acoustic startle assay with precise kinematic tracking further revealed that BMAA, AEG, and 2,4-DAB all impair startle performance. We have previously shown that BMAA increases the frequency of Mauthner-cell-dependent shortlatency C-bends (SLCs) in larval zebrafish (Martin et al. 2021), and our current data reinforce that finding (Fig. 4A), indicating that the startle circuit is made hyperexcitable by BMAA. The isomer AEG also elicited the same hypersensitivity phenotype, and both BMAA and AEG decreased startle latency, suggesting they may act through similar mechanisms. Supporting this possibility, we also found that the binary mixture of BMAA and AEG produced the same hypersensitivity and latency phenotypes (Fig. 4B, Supplementary Fig. 2A), indicating that they likely interact in an additive manner. AEG has been shown to activate metabotropic glutamate receptor 5 (mGluR5) (Schneider et al. 2020), and BMAA can also act through mGluRs as well as NMDA receptors (Chiu et al. 2012; Pierozan and Karlsson 2019). Thus, there is strong evidence that both BMAA and AEG exert their neurotoxic effects by agonizing glutamate receptors.

Although 2,4-DAB can also agonize NMDA receptors and depolarize neurons (Spasic et al. 2018), we did not observe the same hypersensitive startle phenotype in 2,4-DABexposed zebrafish larvae. Rather, we observed that 2,4-DAB modulates startle kinematics, suggesting that 2,4-DAB acts through distinct mechanisms to cause neurotoxicity. The performance of SLC responses, defined through kinematic parameters such as curvature, angular velocity, and duration, depends on the proper function of the underlying motor circuits in the hindbrain and spinal cord, along with that of axial muscles. If startle kinematic defects are observed, this is an indication of motor dysfunction (Spasic et al. 2018). As 2,4-DAB did not alter the frequency or latency of SLCs, in contrast to BMAA and AEG, but instead impacted curvature, angular velocity, and duration (Fig. 5), 2,4-DAB likely acts downstream of the command-like Mauthner neurons. In a recent forward genetic screen in larval zebrafish, several genes were identified that regulate the magnitude of the startle response, with mutations in prdm12b, dolk, and kcnala causing a similar increase in body curvature (Meserve et al. 2021) to that seen in our 2,4-DAB-exposed larvae. Dolichol kinase (dolk), a key enzyme in the protein glycosylation pathway, can regulate the hindbrain expression of the potassium Shaker-like channel encoded by kcnala (Meserve et al. 2021), and mutations in dolk have been identified in patients with neuropathological conditions such as seizures (Helander et al. 2013) and autism spectrum disorder (Xiong et al. 2019). These studies support the idea that 2,4-DAB impacts similar molecular pathways to cause neurotoxicity.

Our unbiased proteomics analysis following in vivo 2,4$\mathrm{DAB}$ exposure, to the best of our knowledge the first such dataset, further implicates 2,4-DAB in neurotoxic processes. We found that at both $2 \mathrm{dpf}$ and $4 \mathrm{dpf} 2,4-\mathrm{DAB}$ strongly 
impacts metabolic function (Fig. 6C), and consistent with the exaggerated startle phenotype caused by $2,4-\mathrm{DAB}$ and shared with dolk mutants, 2,4-DAB inhibited glycosylation (Figs. 6D and 7B). We also found that 2,4-DAB strongly disrupts several canonical pathways related to protein damage, including those associated with the NRF2-mediated oxidative stress response, the unfolded protein response, and endoplasmic reticulum stress (Fig. 6D). These pathways have been implicated in neurodegeneration, which often features an increased incidence of misfolded proteins within neurons (Zhang et al. 2018). Interestingly, disruption of molecules involved in these pathways has also been observed in BMAA-exposed zebrafish (Frøyset et al. 2016). Moreover, the NRF2 oxidative stress response and the nitric oxide synthase (NOS) signaling pathways play important roles in maintaining cellular redox homeostasis and are implicated in ALS pathogenesis (Steinert et al. 2010; Tripathi et al. 2020). We found that 2,4-DAB-induced activation of the NOS signaling pathway was significant after just 2 days of exposure and was further enhanced with continued exposure at 4 days. Disruption of these pathways indicates the likelihood of protein damage due to 2,4-DAB exposure. For example, the nitric oxide radical is a known intracellular secondary messenger that can also react with superoxide anions to form peroxynitrite, which is a highly reactive molecule that can cause irreversible cell damage (Böhme et al. 1993). Consistent with our results, a previous study found that downregulation of NRF2 promotes increased levels of redox species and reduced expression of SODn1, a NRF2-dependent gene (Zou et al. 2016). We also observed significant downregulation of wild-type SOD1 and NFE2L2, and both SOD1 and NFE2L2 have been strongly associated with neurodegeneration (von Otter et al. 2010; Baskoylu et al. 2018). Further supporting a link between 2,4-DAB and motor neuron disease, the ALS-associated gene Ubiquilin 4 (UBQLN4) was among the top ten most strongly downregulated proteins in our 2,4-DAB-exposed samples. Ubiquilin 4 regulates autophagy and protein degradation, and expression of an ALS patientderived gene variant in mouse motor neurons and zebrafish altered motor axon morphology (Edens et al. 2017). Thus, the molecular perturbations induced by $2,4-\mathrm{DAB}$ exposure align with our behavior results and are consistent with neurodegenerative processes.

\section{Conclusions}

Altogether, our findings provide new evidence that 2,4-DAB drives toxicity in zebrafish larvae. Although BMAA, AEG, and 2,4-DAB all show signs of both toxicity and neurotoxicity in larval zebrafish, 2,4-DAB alone causes both increased mortality and motor dysfunction. Finally, developmental exposure to 2,4-DAB affects zebrafish protein homeostasis and biological processes related to neurodegeneration. These results highlight the need for further investigation into 2,4DAB's role in disease and the specific mechanisms by which it exerts its effects.

Abbreviations SLC: Short-latency c startle; LLC: Long latency c startle; HPLC: High-performance liquid chromatography; LC/ MS: High-pressure liquid chromatography combined mass spectrometry; IPA: Ingenuity pathway analysis; ANOVA: Analysis of variance; GO: Gene ontology; BMAA: $\beta$-Methylamino-L-alanine; AEG: N-(2-aminoethyl)glycine; 2,4-DAB: 2,4-Diaminobutyric acid; DEPs: Differentially expressed proteins

Supplementary Information The online version contains supplementary material available at https://doi.org/10.1007/s12640-021-00465-4.

Acknowledgements We are thankful for startup funds provided by North Carolina State University (NCSU), support from the National Institute of Neurological Disorders and Stroke (R01 NS116354), and for pilot project support from the Center for Human Health and Environment (P30 ES025128). We are grateful to Derek Burton, MSc, for zebrafish care and technical support and to Dr. Jeffrey Enders, Ph.D., for feedback on the manuscript. The proteomics section of this work was performed using instrumentation from the Molecular Education, Technology and Research Innovation Center (METRIC) at NC State University.

\section{Declarations}

Ethics Approval Animal experiments conform to internationally accepted standards and have been approved by the Institutional Animal Care and Use Committee (IACUC) at the University of North Carolina State University.

Competing Interest The authors declare no competing interests.

Open Access This article is licensed under a Creative Commons Attribution 4.0 International License, which permits use, sharing, adaptation, distribution and reproduction in any medium or format, as long as you give appropriate credit to the original author(s) and the source, provide a link to the Creative Commons licence, and indicate if changes were made. The images or other third party material in this article are included in the article's Creative Commons licence, unless indicated otherwise in a credit line to the material. If material is not included in the article's Creative Commons licence and your intended use is not permitted by statutory regulation or exceeds the permitted use, you will need to obtain permission directly from the copyright holder. To view a copy of this licence, visit http://creativecommons.org/licenses/by/4.0/.

\section{References}

Atkin G, Paulson H. (2014) Ubiquitin pathways in neurodegenerative disease. Front Mol Neurosci Jul 8;7:63. https://doi.org/10.3389/fnmol. 2014.00063. PMID: 25071440; PMCID: PMC4085722

Banack SA, Cox PA (2003) Biomagnification of cycad neurotoxins in flying foxes: implications for ALS-PDC in Guam. Neurology 61(3):387-389

Baskoylu SN, Yersak J, O’Hern P, Grosser S, Simon J, Kim S, Schuch K, Dimitriadi M, Yanagi KS, Lins J, Hart AC (2018) Single copy/knock-in models of ALS SOD1 in C. elegans suggest 
loss and gain of function have different contributions to cholinergic and glutamatergic neurodegeneration. PLoS Genet 14(10):e1007682-e1007682

Beri J, Nash T, Martin RM, Bereman MS (2017) Exposure to BMAA mirrors molecular processes linked to neurodegenerative disease. Proteomics 17(17-18):1700161

Böhme GA, Bon C, Lemaire M, Reibaud M, Piot O, Stutzmann JM, Doble A, Blanchard JC (1993) Altered synaptic plasticity and memory formation in nitric oxide synthase inhibitor-treated rats. Proc Natl Acad Sci USA 90(19):9191-9194

Brettschneider J, Van Deerlin VM, Robinson JL, Kwong L, Lee EB, Ali YO, Safren N, Monteiro MJ, Toledo JB, Elman L, McCluskey L, Irwin DJ, Grossman M, Molina-Porcel L, Lee VM, Trojanowski JQ (2012) Pattern of ubiquilin pathology in ALS and FTLD indicates presence of C9ORF72 hexanucleotide expansion. Acta Neuropathol 123(6):825-839

Burgess HA, Granato M (2007a) Modulation of locomotor activity in larval zebrafish during light adaptation. J Exp Biol 210(Pt 14):2526-2539

Burgess HA, Granato M (2007b) Sensorimotor gating in larval zebrafish. J Neurosci off J Soc Neurosci 27(18):4984-4994

Caller TA, Doolin JW, Haney JF, Murby AJ, West KG, Farrar HE, Ball A, Harris BT, Stommel EW (2009) A cluster of amyotrophic lateral sclerosis in New Hampshire: a possible role for toxic cyanobacteria blooms. Amyotroph Lateral Scler 10(S2):101-108

Chatziefthimiou AD, Chatziefthimiou AD, Deitch EJ, Deitch EJ, Glover WB, Glover WB, Powell JT, Powell JT, Banack SA, Banack SA, Richer RA, Richer RA, Cox PA, Cox PA, Metcalf JS, Metcalf JS (2018) Analysis of neurotoxic amino acids from marine waters, microbial mats, and seafood destined for human consumption in the Arabian Gulf. Neurotox Res 33(1):143-152

Chiu AS, Braidy N, Marçal H, Welch JH, Gehringer MM, Guillemin GJ, Neilan BA (2015) Global cellular responses to $\beta$-methylamino-l-alanine (BMAA) by olfactory ensheathing glial cells (OEC). Toxicon 99:136-145

Chiu AS, Gehringer MM, Braidy N, Guillemin GJ, Welch JH, Neilan BA (2012) Excitotoxic potential of the cyanotoxin $\beta$-methylamino-1-alanine (BMAA) in primary human neurons. Toxicon 60(6):1159-1165

Chiu AS, Gehringer MM, Braidy N, Guillemin GJ, Welch JH, Neilan BA (2013) Gliotoxicity of the cyanotoxin, $\beta$-methyl-amino-Lalanine (BMAA). Sci Rep 3(1):1482-1482

Cornell JA (2011) A retrospective view of mixture experiments. Qual Eng 23(4):315-331

Cox PA, Banack SA, Murch SJ, Rasmussen U, Tien G, Bidigare RR, Metcalf JS, Morrison LF, Codd GA, Bergman B, Bowers WS (2005) Diverse taxa of cyanobacteria produce $\beta$-N-methylaminoL-alanine, a neurotoxic amino acid. Proc Natl Acad Sci USA 102(14):5074-5078

Edens BM, Yan J, Miller N, Deng H-X, Siddique T, Ma YC (2017) A novel ALS-associated variant in UBQLN4 regulates motor axon morphogenesis. eLife 6

Faassen EJ (2014) Presence of the neurotoxin BMAA in aquatic ecosystems: What do we really know? Toxins 6(3):1109-1138

Ferraiuolo RM, Meister D, Leckie D, Dashti M, Franke J, Porter LA, Trant JF (2019) Neuro- and hepatic toxicological profile of (S)2,4-diaminobutanoic acid in embryonic, adolescent and adult zebrafish. J Appl Toxicol 39(11):1568-1577

Field NC, Metcalf JS, Caller TA, Banack SA, Cox PA, Stommel EW (2013) Linking $\beta$-methylamino-l-alanine exposure to sporadic amyotrophic lateral sclerosis in Annapolis, MD. Toxicon 70:179-183

Frøyset AK, Khan EA, Fladmark KE (2016) Quantitative proteomics analysis of zebrafish exposed to sub-lethal dosages of $\beta$-methylamino-L-alanine (BMAA). Sci Rep 6:29631
Groten JP, Feron VJ, Sühnel J (2001) Toxicology of simple and complex mixtures. Trends Pharmacol Sci 22:316-322

Helander A, Stödberg T, Jaeken J, Matthijs G, Eriksson M, Eggertsen G (2013) Dolichol kinase deficiency (DOLK-CDG) with a purely neurological presentation caused by a novel mutation. Mol Genet Metab 110(3):342-344

Jungblut AD, Wilbraham J, Banack SA, Metcalf JS, Codd GA (2018) Microcystins, BMAA and BMAA isomers in 100-year-old Antarctic cyanobacterial mats collected during Captain R.F. Scott's Discovery Expedition. Eur J Phycol 53(2):115

Karalis V, Bateup HS (2021) Current approaches and future directions for the treatment of mTORopathies. Dev Neurosci 43(3-4):143-158

Karamyan VT, Speth RC (2008) Animal models of BMAA neurotoxicity: a critical review. Life Sci 82(5):233-246

Khatri P, Drăghici S (2005) Ontological analysis of gene expression data: current tools, limitations, and open problems. Bioinformatics (Oxford, England) 21(18):3587-3595

Krämer A, Green J, Pollard JJ, Tugendreich S (2014) Causal analysis approaches in ingenuity pathway analysis. Bioinformatics (Oxford, England) 30(4):523-530

Lance E, Arnich N, Maignien T, Biré R (2018) Occurrence of $\beta$-Nmethylamino-l-alanine (BMAA) and isomers in aquatic environments and aquatic food sources for humans. Toxins 10(2):83

Li B, Yu S, Li G, Chen X, Huang M, Liao X, Li H, Hu F, Wu J (2019) Transfer of a cyanobacterial neurotoxin, $\beta$-methylamino-1-alanine from soil to crop and its bioaccumulation in Chinese cabbage. Chemosphere (Oxford) 219:997-1001

Lobner D (2009) Mechanisms of beta-N-methylamino-L-alanine induced neurotoxicity. Amyotroph Lateral Scler 10(Suppl 2):56-60

Main BJ, Main BJ, Rodgers KJ, Rodgers KJ (2018) Assessing the combined toxicity of BMAA and its isomers 2,4-DAB and AEG in vitro using human neuroblastoma cells. Neurotox Res 33(1):33-42

Marquart GD, Tabor KM, Bergeron SA, Briggman KL, Burgess HA (2019) Prepontine non-giant neurons drive flexible escape behavior in zebrafish. PLoS Biol 17(10):e3000480-e3000480

Marsden KC, Jain RA, Wolman MA, Echeverry FA, Nelson JC, Hayer KE, Miltenberg B, Pereda AE, Granato M (2018) A Cyfip2dependent excitatory interneuron pathway establishes the innate startle threshold. Cell Rep 23(3):878-887

Martin RM, Bereman MS, Marsden KC (2020) BMAA and MCLR interact to modulate behavior and exacerbate molecular changes related to neurodegeneration in larval zebrafish. Toxicol Sci 179(2):251-261

Martin RM, Bereman MS, Marsden KC (2021) BMAA and MCLR Interact to Modulate Behavior and Exacerbate Molecular Changes Related to Neurodegeneration in Larval Zebrafish. Toxicol Sci Jan 28;179(2):251-261. https://doi.org/10.1093/toxsci/kfaa178. PMID: 33295630; PMCID: PMC8502428

Martin RM, Stallrich J, Bereman MS (2019) Mixture designs to investigate adverse effects upon co-exposure to environmental cyanotoxins. Toxicology 421:74-83

Masseret E, Banack S, Boumédiène F, Abadie E, Brient L, Pernet F, Juntas-Morales R, Pageot N, Metcalf J, Cox P, Camu W, the French Network on A. L. S. C. D. and Investigation (2013) Dietary BMAA exposure in an amyotrophic lateral sclerosis cluster from southern France. PLoS One 8(12):e83406-e83406

Mattson MP (2006) Neuronal life-and-death signaling, apoptosis, and neurodegenerative disorders. Antioxid Redox Signal 8(11-12):1997-2006

Meserve JH, Nelson JC, Marsden KC, Hsu J, Echeverry FA, Jain RA, Wolman MA, Pereda AE, Granato M (2021) A forward genetic screen identifies Dolk as a regulator of startle magnitude 
through the potassium channel subunit Kv1.1. PLoS Genet 17(6):e1008943-e1008943

Metcalf JS, Banack SA, Lindsay J, Morrison LF, Cox PA, Codd GA (2008) Co-occurrence of $\beta$-N-methylamino-l-alanine, a neurotoxic amino acid with other cyanobacterial toxins in British waterbodies, 1990-2004. Environ Microbiol 10:702-708

Metcalf JS, Richer R, Cox PA, Codd GA (2012) Cyanotoxins in desert environments may present a risk to human health. Sci Total Environ 421-422:118-123

Murakami K, Murata N, Noda Y, Tahara S, Kaneko T, Kinoshita N, Hatsuta H, Murayama S, Barnham KJ, Irie K, Shirasawa T, Shimizu T (2011) SOD1 (copper/zinc superoxide dismutase) deficiency drives amyloid $\beta$ protein oligomerization and memory loss in mouse model of Alzheimer disease. J Biol Chem 286(52):44557-44568

Nunes-Costa D, Magalhães JD, G-Fernandes M, Cardoso SM, Empadinhas N (2020) Microbial BMAA and the pathway for Parkinson's disease neurodegeneration. Front Aging Neurosci 12:26

Politis SN, Colombo P, Colombo G, Rekkas DM (2017) Design of experiments (DoE) in pharmaceutical development. Drug Dev Ind Pharm 43(6):889-901

Pierozan P, Karlsson O (2019) Mitotically heritable effects of BMAA on striatal neural stem cell proliferation and differentiation. Cell Death Dis 10(7):478-414

Pomati F, Orlandi C, Clerici M, Luciani F, Zuccato E (2008) Effects and interactions in an environmentally relevant mixture of pharmaceuticals. Toxicol Sci 102(1):129-137

Purdie EL, Samsudin S, Eddy FB, Codd GA (2009) Effects of the cyanobacterial neurotoxin $\beta$ - N-methylamino-L-alanine on the early-life stage development of zebrafish ( Danio rerio). Aquat Toxicol 95(4):279-284

Réveillon D, Abadie E, Séchet V, Masseret E, Hess P, Amzil Z (2015) $\beta$-N-methylamino-l-alanine (BMAA) and isomers: distribution in different food web compartments of Thau lagoon, French Mediterranean Sea. Mar Environ Res 110:8-18

Réveillon D, Séchet V, Hess P, Amzil Z (2016) Production of BMAA and DAB by diatoms (Phaeodactylum tricornutum, Chaetoceros sp., Chaetoceros calcitrans and Thalassiosira pseudonana) and bacteria isolated from a diatom culture. Harmful Algae 58:45-50

Rush T, Liu X, Lobner D (2012) Synergistic toxicity of the environmental neurotoxins methylmercury and $\beta$-N-methylamino-Lalanine. NeuroReport 23(4):216-219

Saha S, Buttari B, Panieri E, Profumo E, Saso L (2020) An overview of Nrf2 signaling pathway and its role in inflammation. Molecules 25(22)

Samardzic K, Steele JR, Violi JP, Colville A, Mitrovic SM, Rodgers KJ (2021) Toxicity and bioaccumulation of two non-protein amino acids synthesised by cyanobacteria, $\beta$-N-Methylamino-L-alanine (BMAA) and 2,4-diaminobutyric acid (DAB), on a crop plant. Ecotoxicol Environ Saf 208:111515

Schneider T, Simpson C, Desai P, Tucker M, Lobner D (2020) Neurotoxicity of isomers of the environmental toxin L-BMAA. Toxicon (Oxford) 184:175-179

Scott L, Downing T (2019) Dose-dependent adult neurodegeneration in a rat model after neonatal exposure to $\beta$-N-methylamino-l-alanine. Neurotox Res 35(3):711-723
Scott LL, Downing TG, Timothy D, Laura S (2017) A single neonatal exposure to BMAA in a rat model produces neuropathology consistent with neurodegenerative diseases. Toxins 10(1):22

Silva DF, Candeias E, Esteves AR, Magalhães JD, Ferreira IL, NunesCosta D, Rego AC, Empadinhas N, Cardoso SM (2020) Microbial BMAA elicits mitochondrial dysfunction, innate immunity activation, and Alzheimer's disease features in cortical neurons. J Neuroinflammation 17(1):332

Spasic S, Stanojevic M, Nesovic Ostojic J, Kovacevic S, Prostran M, Lopicic S (2018) Extensive depolarization and lack of recovery of leech Retzius neurons caused by 2,4 diaminobutyric acid. Aquat Toxicol 199:269-275

Steinert JR, Chernova T, Forsythe ID (2010) Nitric oxide signaling in brain function, dysfunction, and dementia. Neuroscientist $16(4): 435-452$

Tripathi MK, Kartawy M, Amal H (2020) The role of nitric oxide in brain disorders: autism spectrum disorder and other psychiatric, neurological, and neurodegenerative disorders. Redox Biol 34:101567-101567

Tyanova S, Temu T, Sinitcyn P, Carlson A, Hein MY, Geiger T, Mann M, Cox J (2016) The Perseus computational platform for comprehensive analysis of (prote)omics data. Nat Methods 13(9):731-740

Violi JP, Mitrovic SM, Colville A, Main BJ, Rodgers KJ (2019) Prevalence of $\beta$-methylamino-L-alanine (BMAA) and its isomers in freshwater cyanobacteria isolated from eastern Australia. Ecotoxicol Environ Saf 172:72-81

Vo Duy S, Munoz G, Dinh QT, Tien Do D, Simon DF, Sauvé S (2019) Analysis of the neurotoxin $\beta$-N-methylamino-L-alanine (BMAA) and isomers in surface water by FMOC derivatization liquid chromatography high resolution mass spectrometry. PLoS One 14(8):e0220698

von Otter M, Landgren S, Nilsson S, Celojevic D, Bergström P, Håkansson A, Nissbrandt H, Drozdzik M, Bialecka M, Kurzawski M, Blennow K, Nilsson M, Hammarsten O, Zetterberg H (2010) Association of Nrf2-encoding NFE2L2 haplotypes with Parkinson's disease. BMC Med Genet 11(1):36-36

Xiong J, Chen S, Pang N, Deng X, Yang L, He F, Wu L, Chen C, Yin F, Peng J (2019) Neurological diseases with autism spectrum disorder: role of ASD risk genes. Front Neurosci 13:349-349

Yi J, Horky LL, Friedlich AL, Shi Y, Rogers JT, Huang X (2009) L-arginine and Alzheimer's disease. Int J Clin Exp Pathol 2(3):211-38. Epub 2008 Oct 2. PMID: 19079617; PMCID: PMC2600464

Zhang Q, Zhang P, Qi G-J, Zhang Z, He F, Lv Z-X, Peng X, Cai H-W, Li T-X, Wang X-M, Tian B (2018) Cdk5 suppression blocks SIRT1 degradation via the ubiquitin-proteasome pathway in Parkinson's disease models. Biochim Biophys Acta 1862(6):1443-1451

Zou Y, Wang J, Peng J, Wei H (2016) Oregano essential oil induces SOD1 and GSH expression through Nrf2 activation and alleviates hydrogen peroxide-induced oxidative damage in IPEC-J2 Cells. Oxid Med Cell Longev 2016:5987183-5987113

Publisher's Note Springer Nature remains neutral with regard to jurisdictional claims in published maps and institutional affiliations. 